\title{
Harnessing bismuth coordination chemistry to achieve bright, long-lived organic phosphorescence
}

\author{
Alexander C. Marwitz, ${ }^{1}$ Aaron D. Nicholas, ${ }^{2}$ Leticia M. Breuer, ${ }^{1}$ Jeffery A. Bertke, ${ }^{1}$ Karah E. Knope ${ }^{1, *}$
}

1 Department of Chemistry, Georgetown University, Washington, D.C. 20057, United States of America

2 National Security Directorate, Pacific Northwest National Laboratory, 902 Battelle Boulevard, Richland, WA

99354, United States of America

*kek44@georgetown.edu

\section{Supporting Information}

I. Crystallographic Refinement Details............................................2

II. Additional Structural Information.......................................................

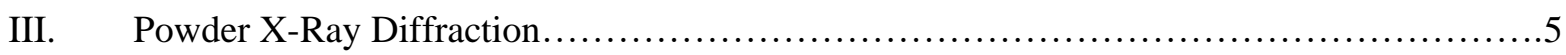

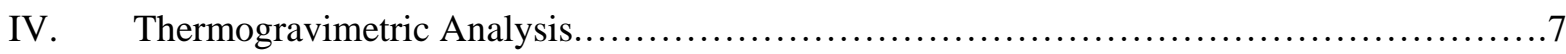

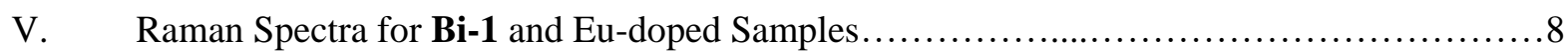

VI. Variable Temperature Luminescence Measurements................................ 10

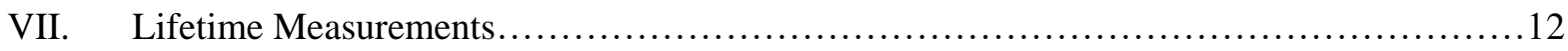

VIII. Diffuse Reflectance and UV-Vis Absorption Spectroscopy ............................20

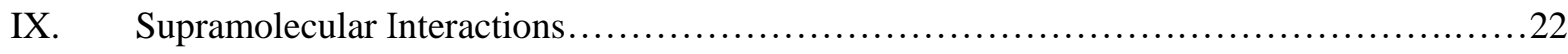

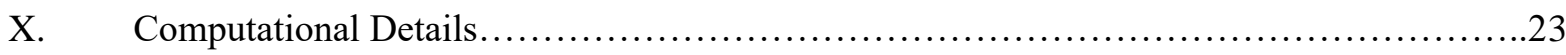

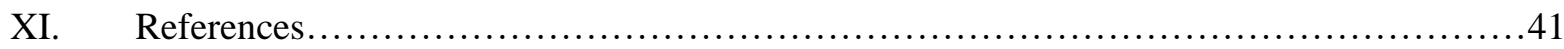




\section{Crystallographic Refinement Details}

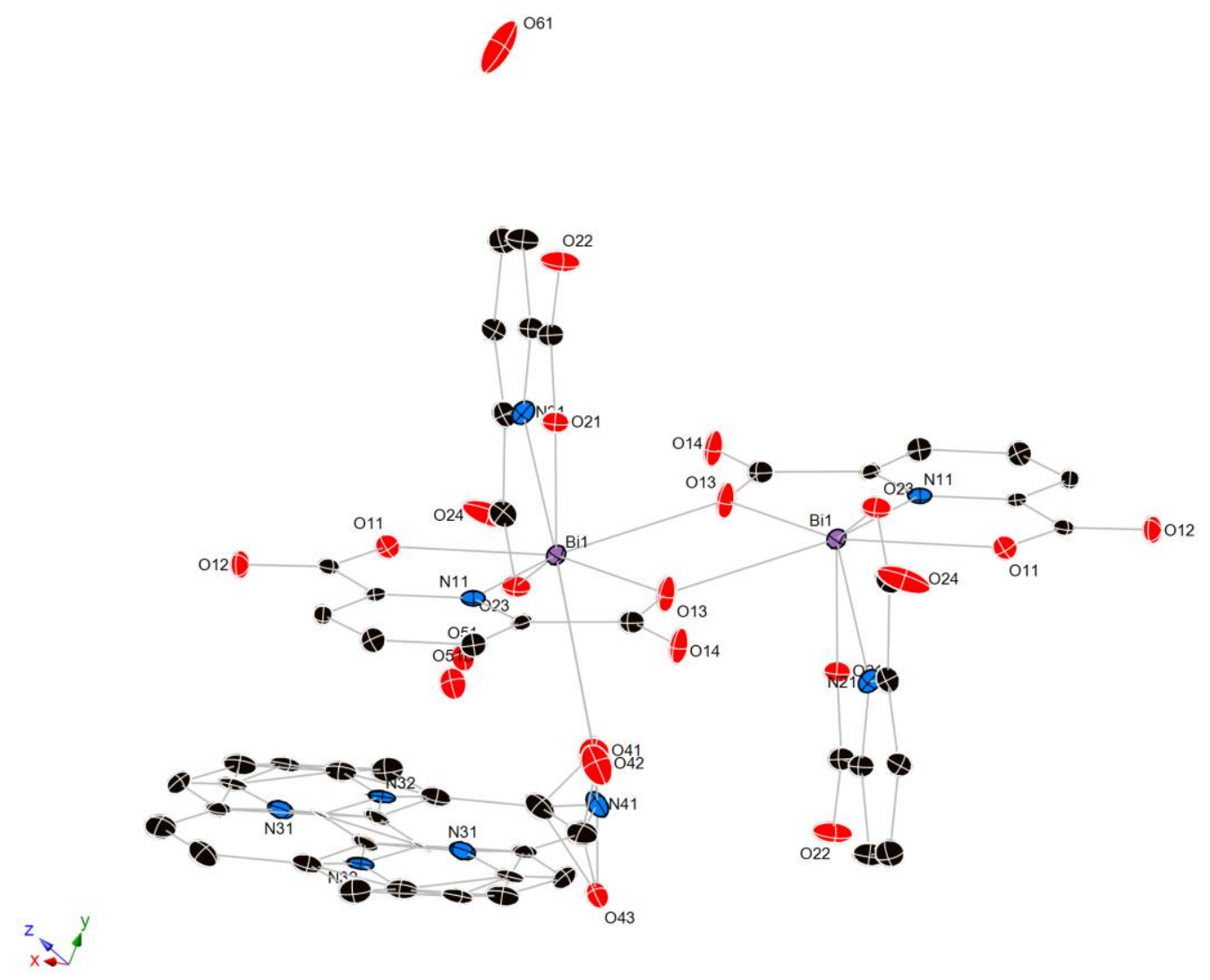

Figure S1. Thermal ellipsoid plot of Bi-1 at $100 \mathrm{~K}$. Ellipsoids are shown at 50\% probability. The disorder of the Phen is shown in the plot. Symmetry operators: (i) $x, y+1, z$.

\section{$\left[\mathrm{Bi}_{2}(\mathrm{HPDC})_{2}(\mathrm{PDC})_{2}\left(\mathrm{NO}_{3}\right)\right] \cdot[\mathrm{Hphen}] \cdot 4 \mathrm{H}_{2} \mathrm{O}(\mathrm{Bi}-1)$}

A structural model consisting of the neutral $\left[\mathrm{Bi}_{2}(\mathrm{PDC})_{2}(\mathrm{HPDC})_{2}\right]$ dimer, one nitrate anion, one 1,10phenanthrolinium cation, and two lattice water molecules was developed. One of the lattice water molecules is disordered over two positions. Similar displacement amplitudes (esd 0.01) were imposed on the disordered oxygen sites overlapping by less than the sum of van der Waals radii. The $(\mathrm{Hphen})\left(\mathrm{NO}_{3}\right)$ ion pair is disordered across a symmetry site and thus refined with negative PART commands.

The water $\mathrm{H}$ atoms could not be located in the difference map and thus were left off of the model. Hydroxyl $\mathrm{H}$ atom positions, $\mathrm{R}-\mathrm{OH}$, were optimized by rotation about $\mathrm{R}-\mathrm{O}$ bonds with idealized $\mathrm{O}-\mathrm{H}$ and R--H distances. Remaining $\mathrm{H}$ atoms were included as riding idealized contributors. Hydroxyl H atom U's were assigned as 1.5 times $\mathrm{U}_{\text {eq }}$ of the carrier atom; remaining $\mathrm{H}$ atom $\mathrm{U}$ 's were assigned as 1.2 times carrier $\mathrm{U}_{\text {eq. }}$ 


\section{Additional Structural Information}

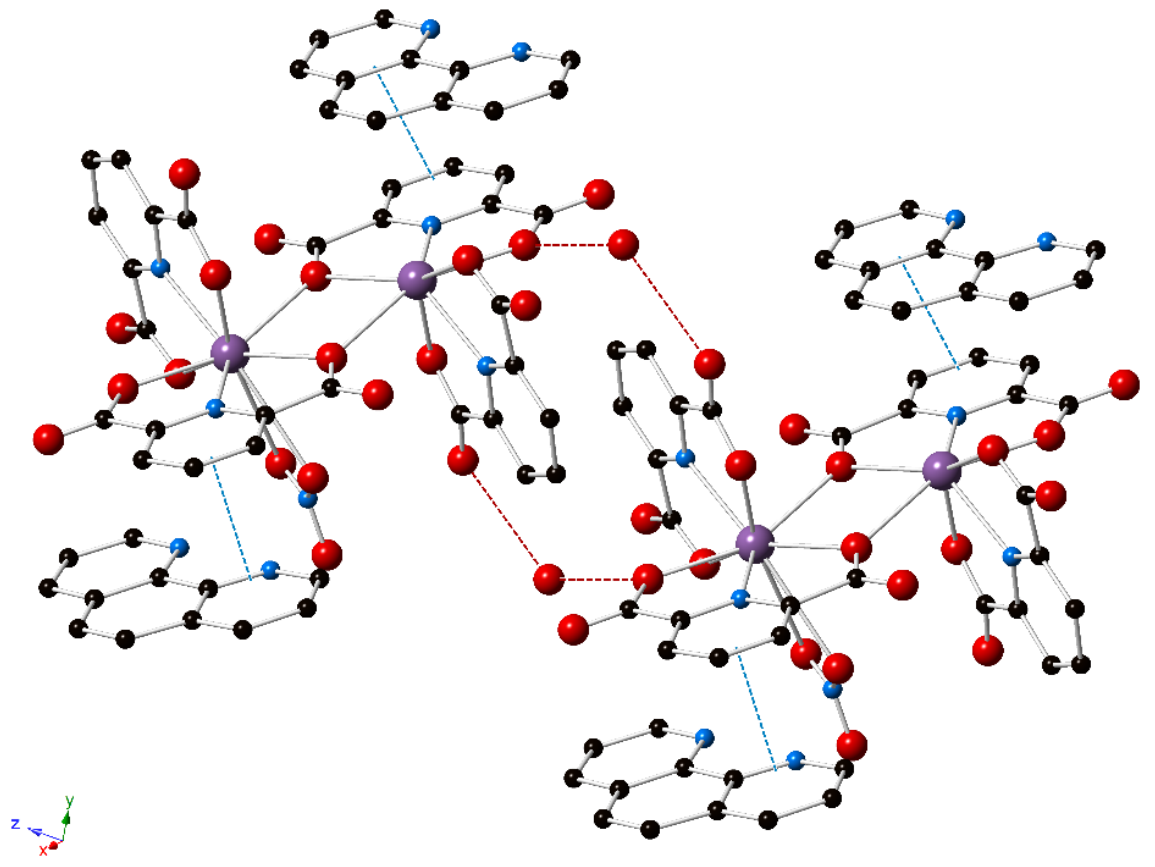

Figure S2. Packing diagram of Bi-1 depicting the hydrogen bonding between PDC ligands and outer coordination sphere waters (red dashed line). $\pi-\pi$ interactions (blue dashed line) are also shown between phenanthroline and PDC ligands. Purple $=$ bismuth, red $=$ oxygen, blue $=$ nitrogen, and black $=$ carbon atoms. Hydrogen atoms have been omitted for clarity.

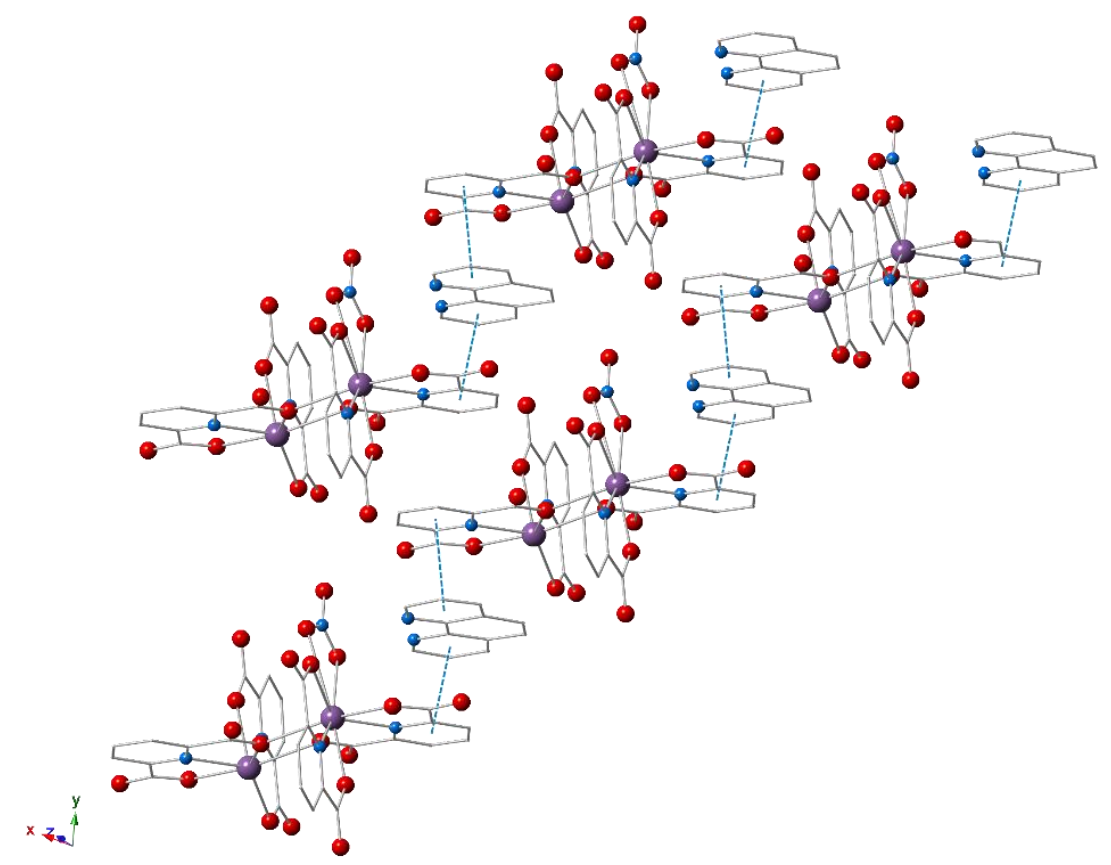

Figure S3. Packing diagram of Bi-1 depicting neighboring 1D-chains of $\pi$-stacking dimers. Hydrogen atoms have been omitted for clarity. 

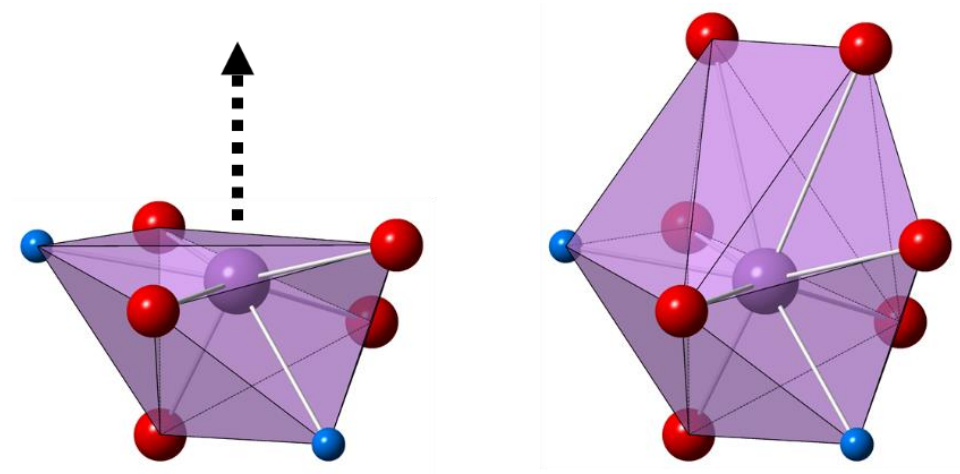

Figure S4. Polyhedral representations of Bi-1 depicting the two possible bismuth coordination environments arising from the 50\% nitrate-Hphen occupancy: a hemi-directed 7-coordinate metal center (left), and a 9-coordinate metal center. The arrow depicts the directionality of the stereochemically active $6 s^{2}$ lone pair. 


\section{Powder X-ray Diffraction}

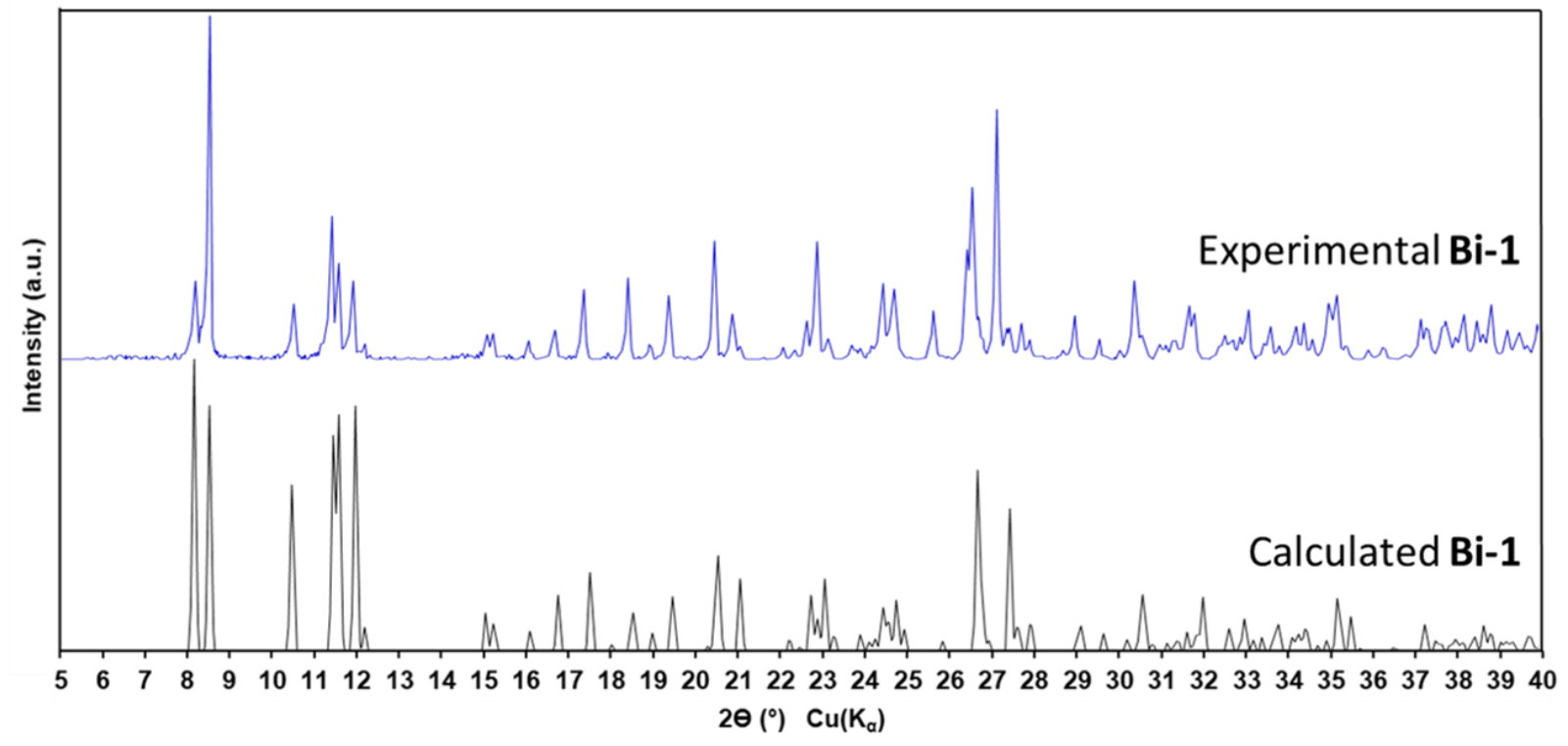

Figure S5. Powder X-ray Diffraction pattern experimentally collected for Bi-1 (blue) overlaid with the pattern calculated from the single crystal data collected at $100 \mathrm{~K}$.

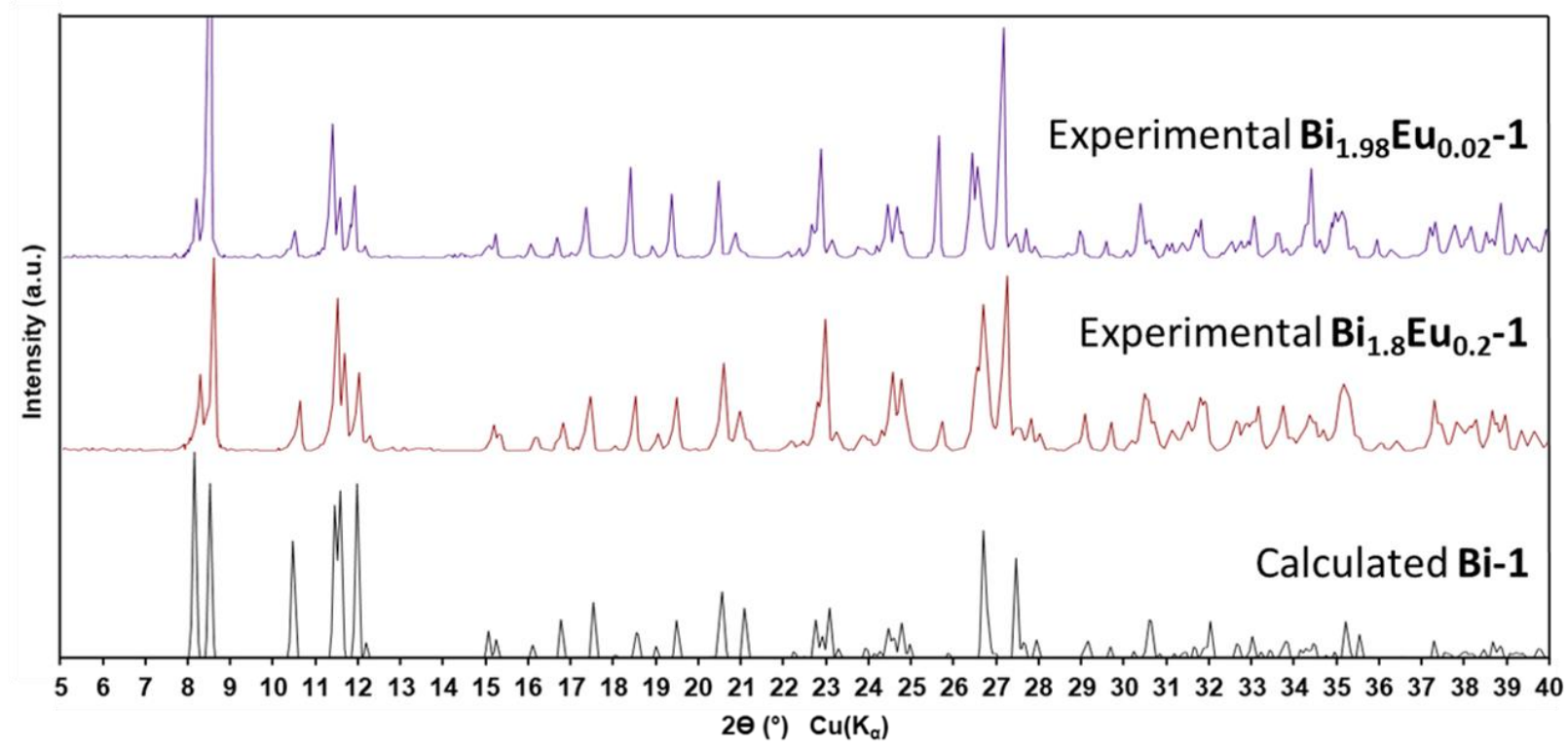

Figure S6. Powder X-ray Diffraction pattern experimentally collected for $\mathbf{B i} \mathbf{i}_{1.98} \mathbf{E} \mathbf{u}_{\mathbf{0 . 0 2}} \mathbf{- 1}$ (purple) and $\mathbf{B i}_{1.8} \mathbf{E u}_{\mathbf{0 . 2}} \mathbf{- 1}$ (red) overlaid with the pattern calculated from the single crystal data for Bi-1 collected at 100 $\mathrm{K}$. 


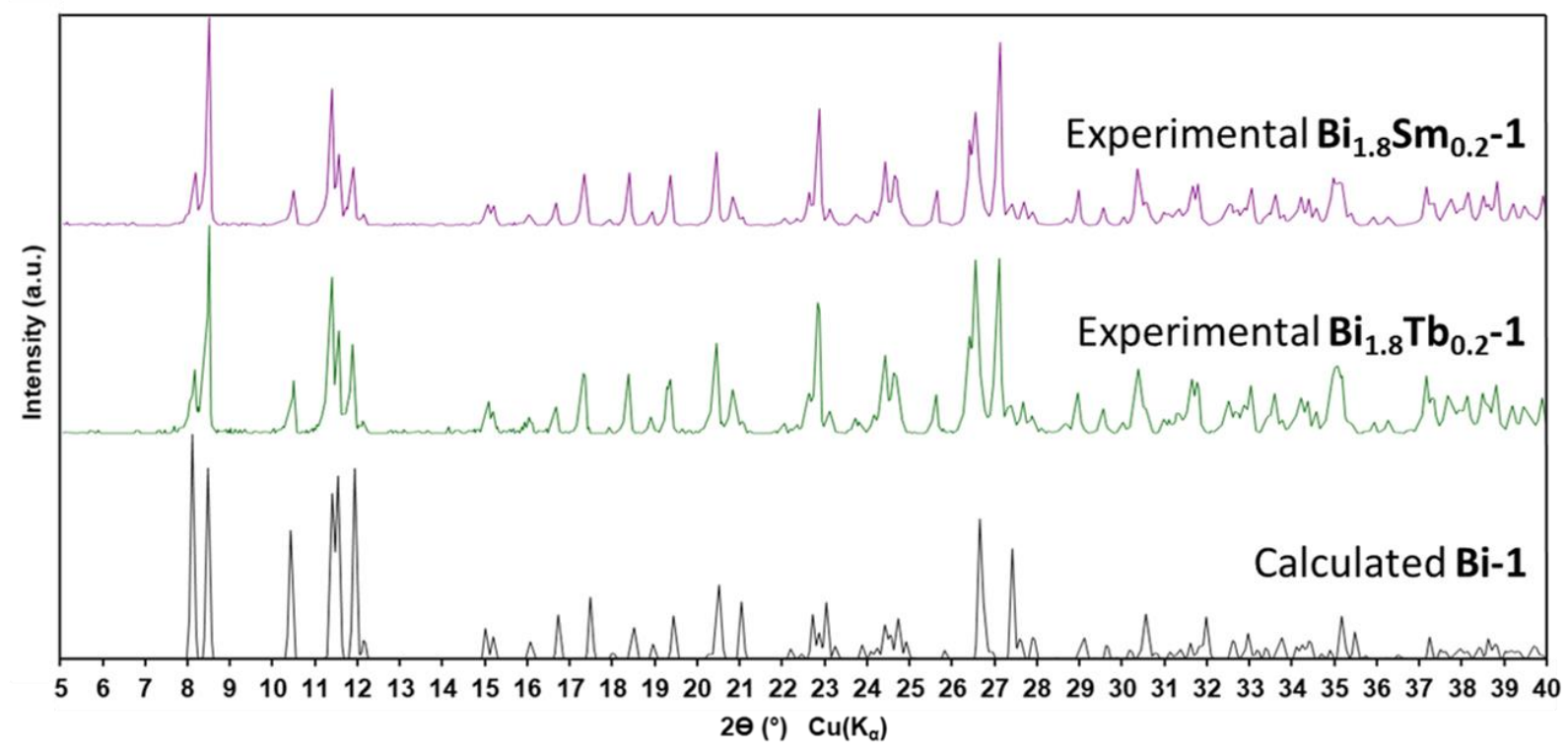

Figure S7. Powder X-ray Diffraction pattern experimentally collected for $\mathbf{B i}_{1.8} \mathbf{S m}_{\mathbf{0 . 2}} \mathbf{- 1}$ (pink) and $\mathbf{B i}_{1.8} \mathbf{T b}_{0.2} \mathbf{1}$ (green) overlaid with the pattern calculated from the single crystal data for $\mathbf{B i}-\mathbf{1}$ collected at $100 \mathrm{~K}$. 


\section{Thermogravimetric Analysis}

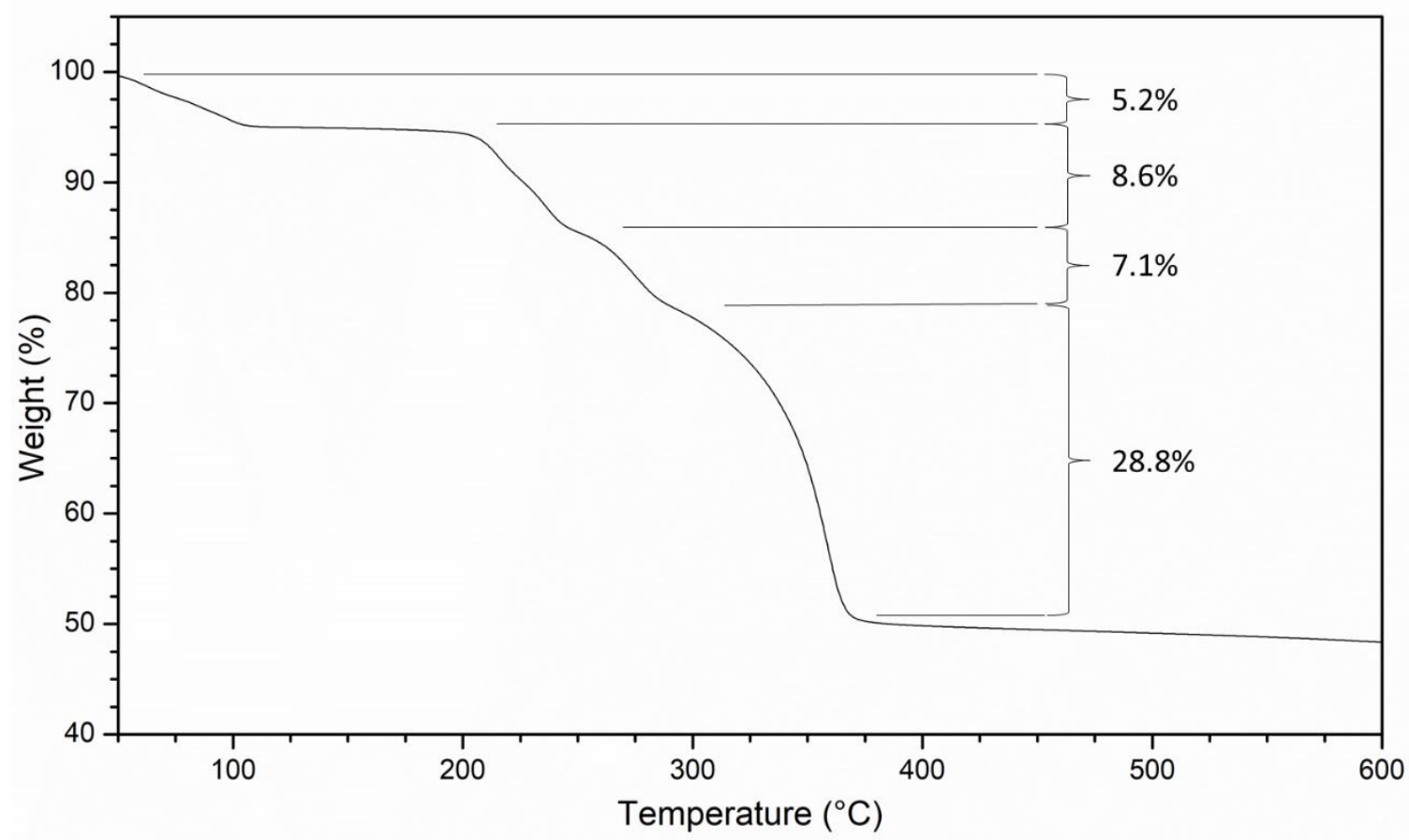

Figure S8. Thermogravimetric analysis of Bi-1 collected from $35-600{ }^{\circ} \mathrm{C}$ at a rate of $5{ }^{\circ} \mathrm{C}$ per minute, with annotations. 


\section{Raman Spectra for Bi-1 and Eu-doped Samples}

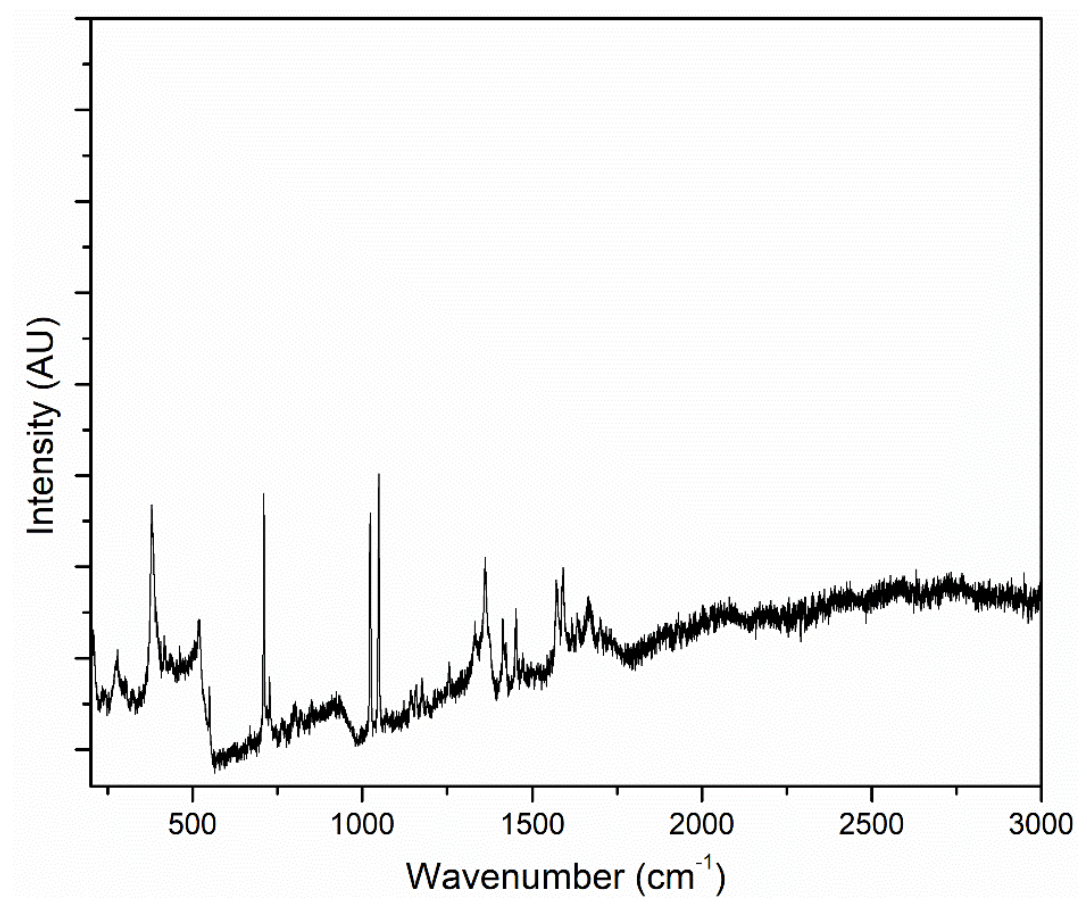

Figure S9. Raman spectrum of Bi-1.

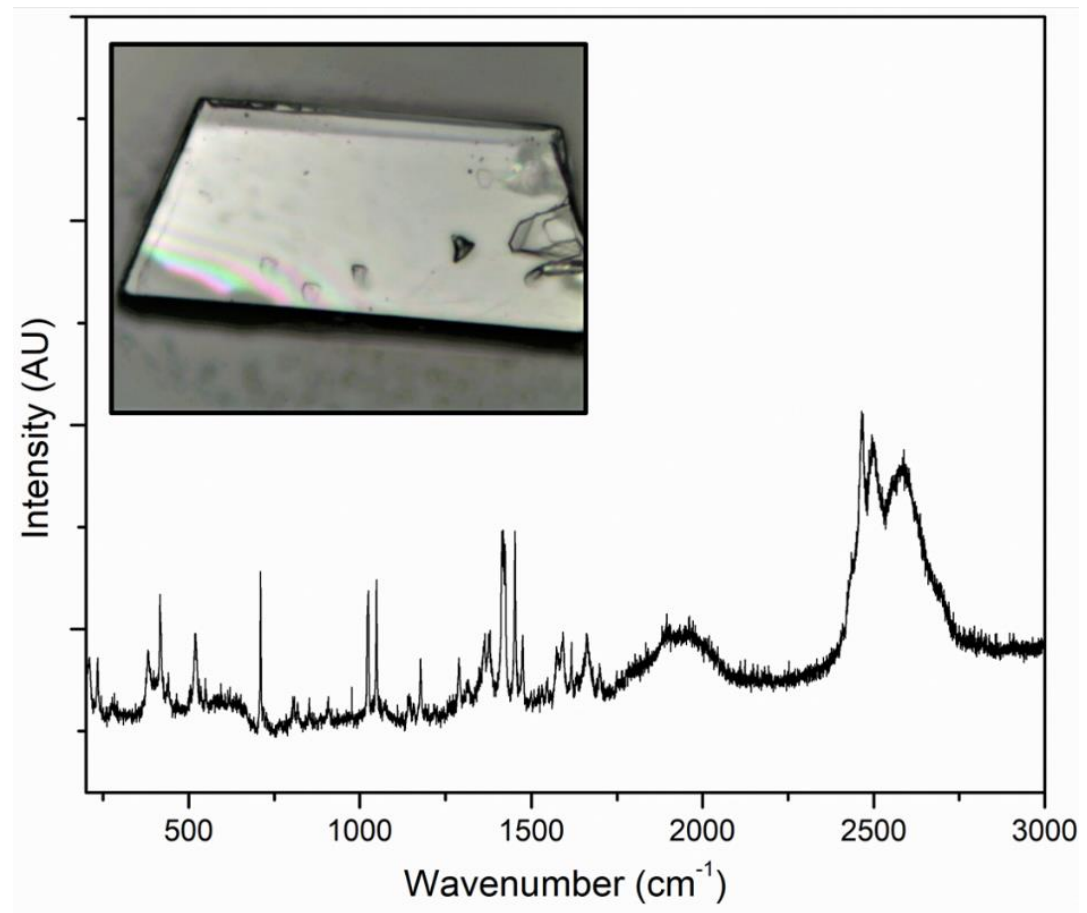

Figure S10. Raman spectrum of $\mathbf{B i}_{1.98} \mathbf{E} \mathbf{u}_{0.02}-1$. The inset shows the single crystal that data was recorded from under a 50x microscope objective. 


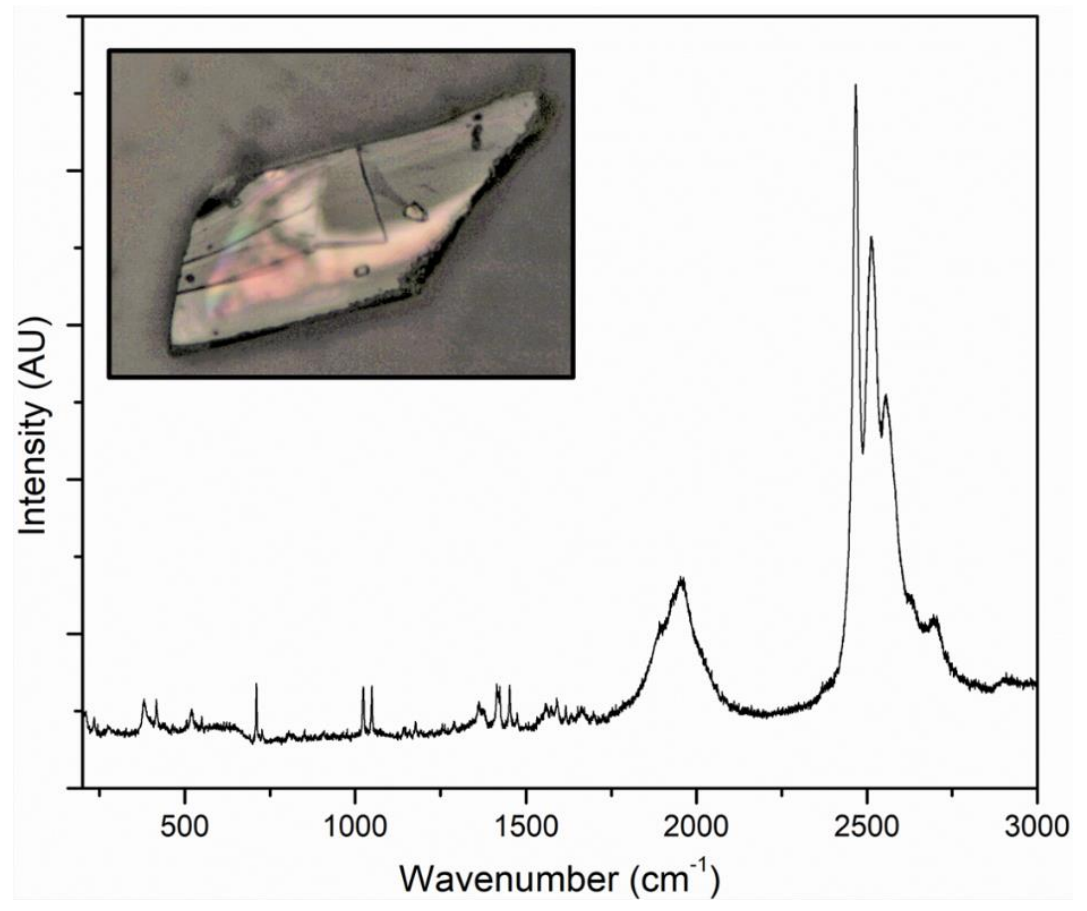

Figure S11. Raman spectrum of $\mathbf{B i}_{1.8} \mathbf{E} \mathbf{u}_{0.2}-1$. The inset shows the single crystal that data was recorded from under a 50x microscope objective.

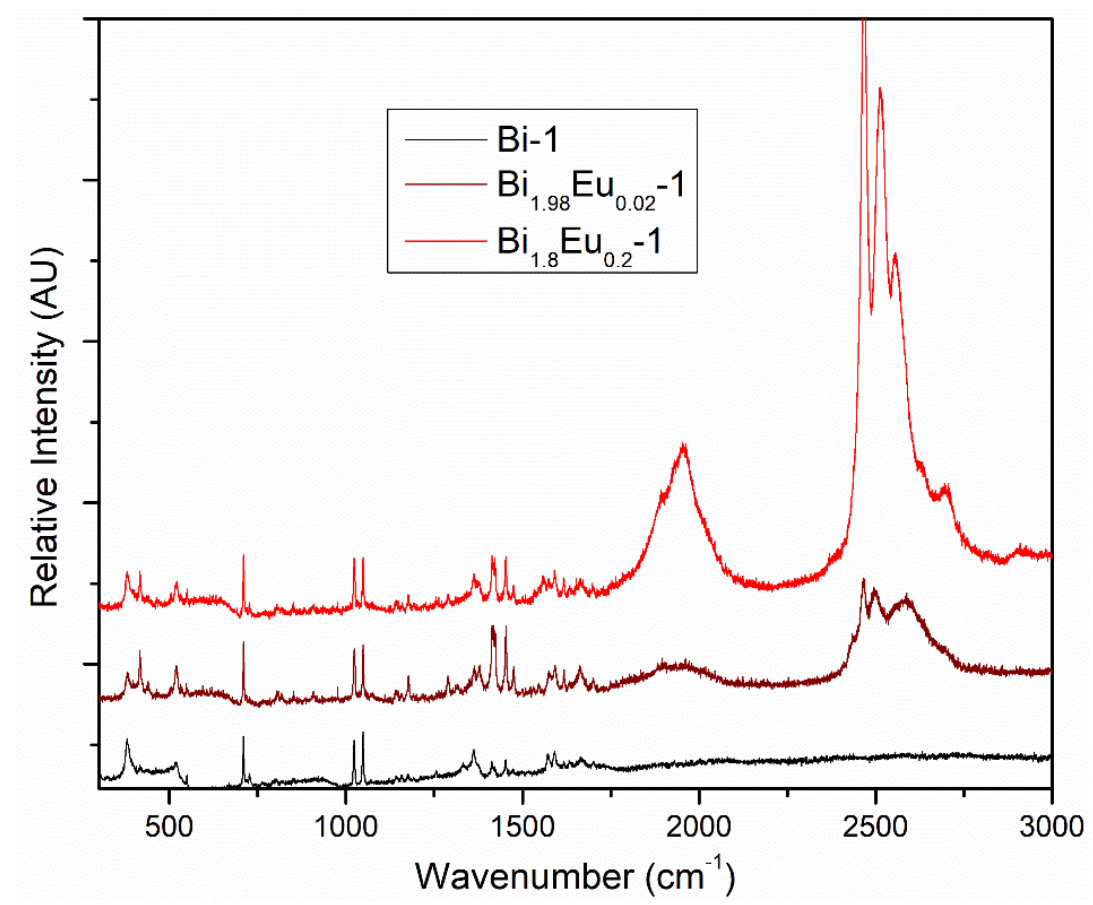

Figure S12. Overlaid raman spectra of $\mathbf{B i - 1 ,} \mathbf{B i}_{1.98} \mathbf{E u}_{0.02}-\mathbf{1}$, and $\mathbf{B i} \mathbf{1}_{\mathbf{1 . 8}} \mathbf{E} \mathbf{u}_{0.2}-\mathbf{1}$. Peak intensity is normalized for peaks around $1000 \mathrm{~cm}^{-1}$ to show the growing peaks due to Eu emission harmonics. 


\section{Variable Temperature Luminescence Measurements}

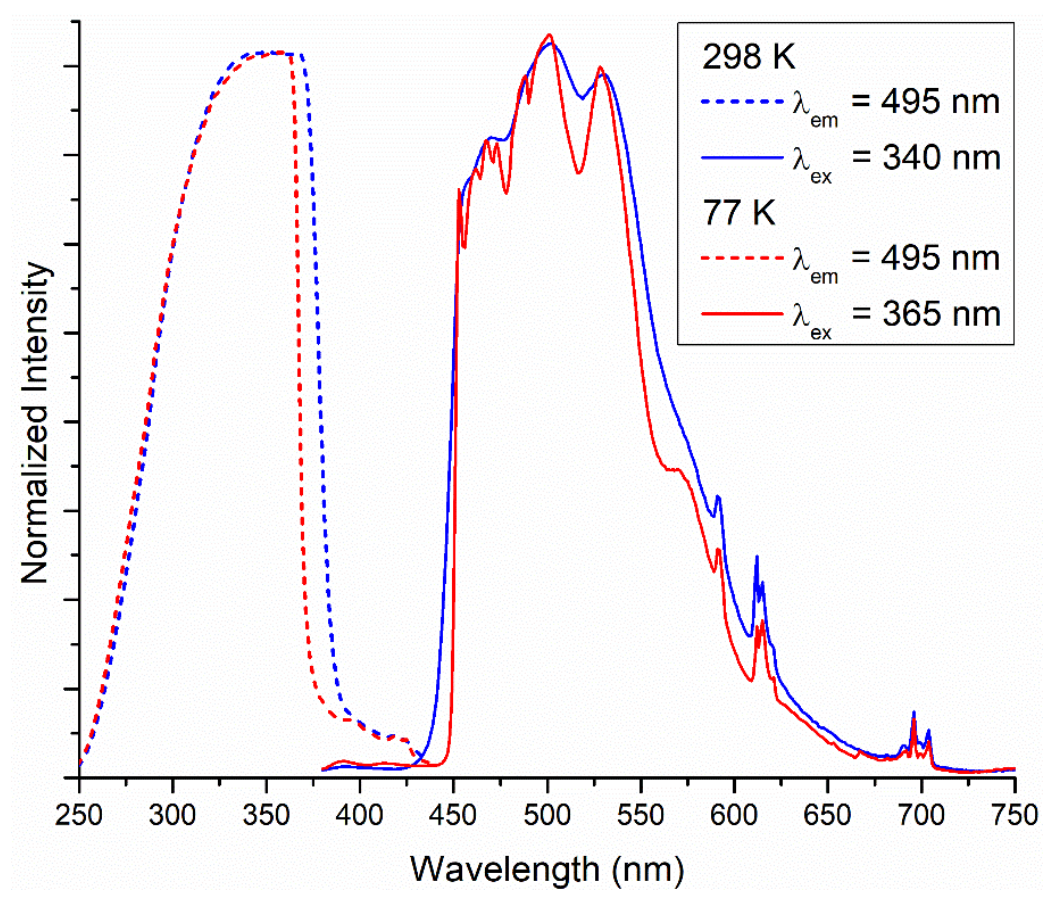

Figure S13. Excitation and emission spectra of $\mathbf{B i}_{1.98} \mathbf{E u}_{0.02}-1$ at $298 \mathrm{~K}$ (blue) and $77 \mathrm{~K}$ (red).

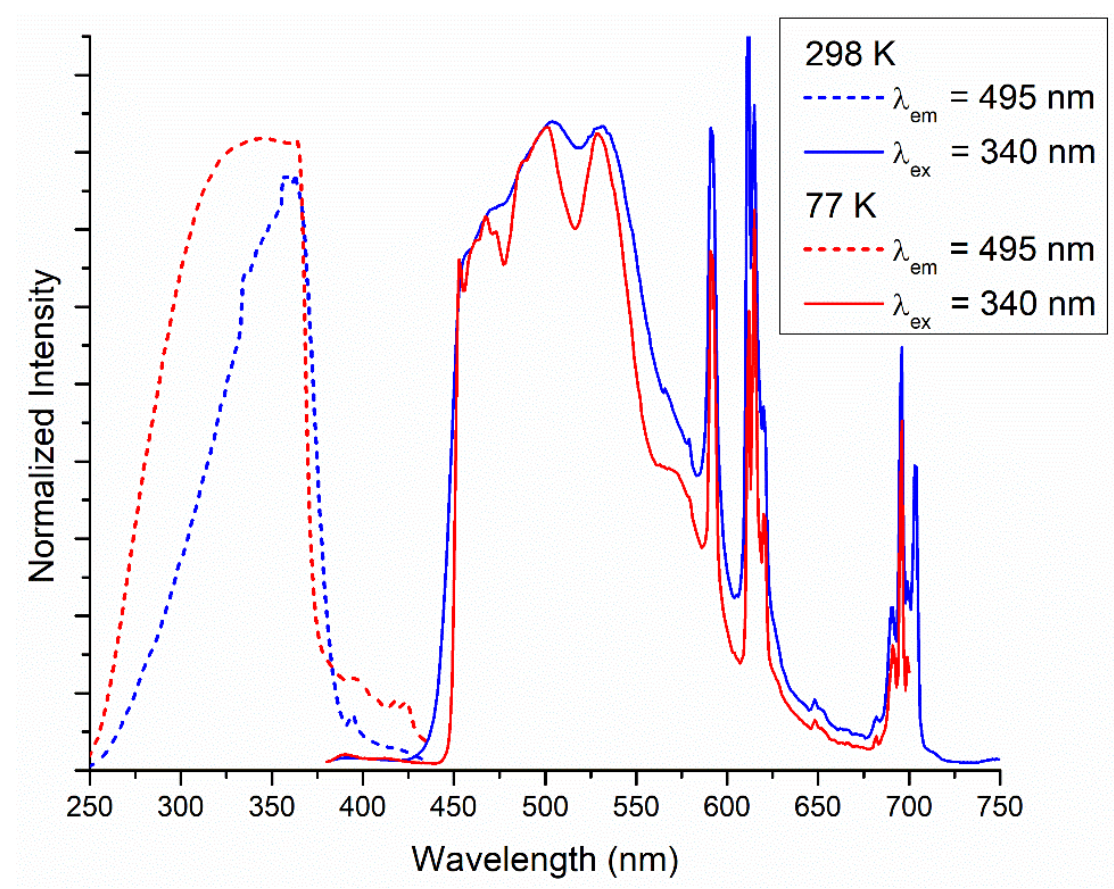

Figure S14. Excitation and emission spectra of $\mathbf{B i}_{1.8} \mathbf{E u}_{0.2} \mathbf{1}$ at $298 \mathrm{~K}$ (blue) and $77 \mathrm{~K}$ (red). 


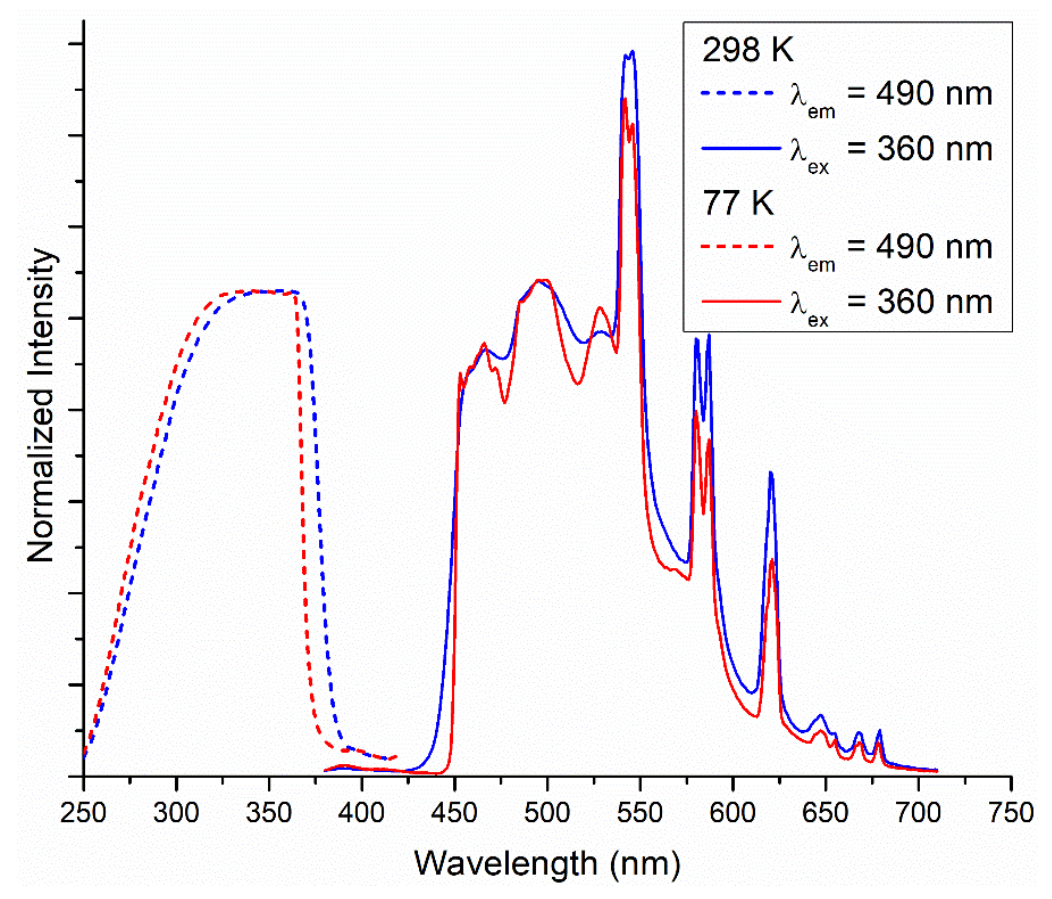

Figure S15. Excitation and emission spectra of $\mathbf{B i}_{1.8} \mathbf{T b}_{\mathbf{0 . 2}} \mathbf{- 1}$ at $298 \mathrm{~K}$ (blue) and $77 \mathrm{~K}$ (red).

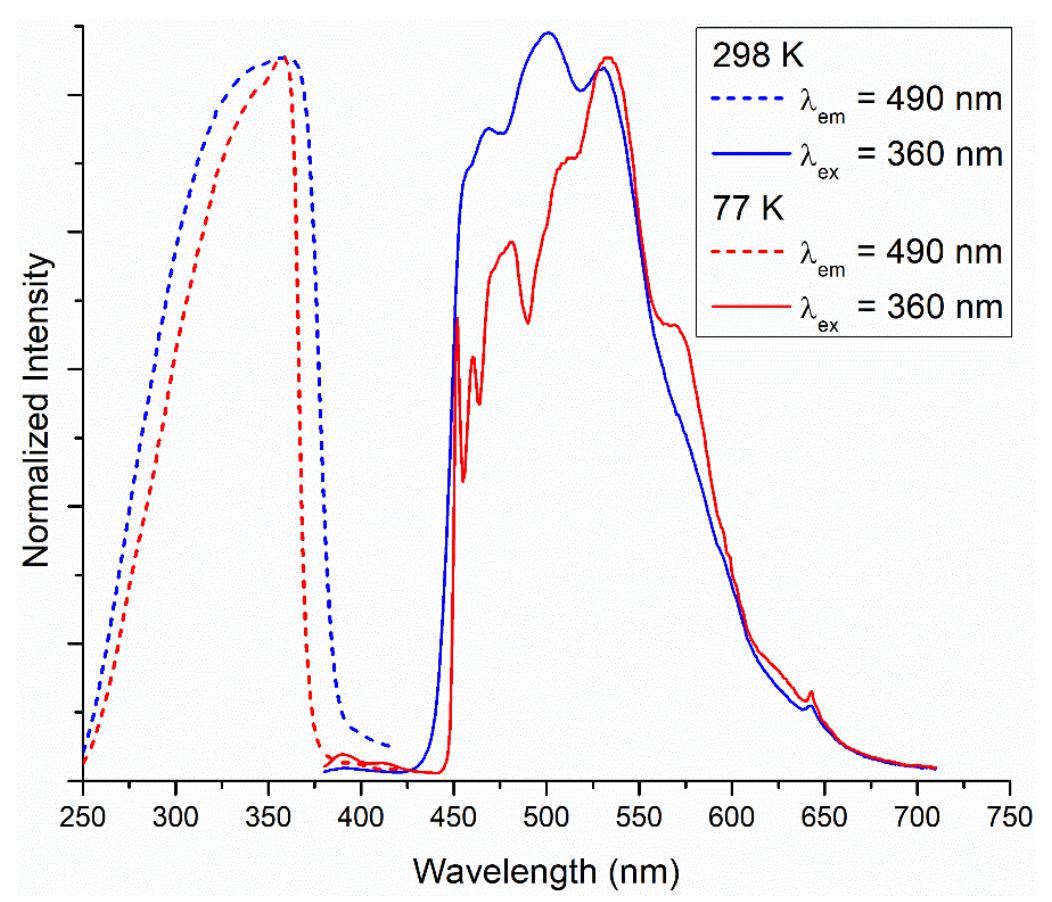

Figure S16. Excitation and emission spectra of $\mathbf{B i}_{1.8} \mathbf{S m}_{0.2} \mathbf{1}$ at $298 \mathrm{~K}$ (blue) and $77 \mathrm{~K}$ (red). 


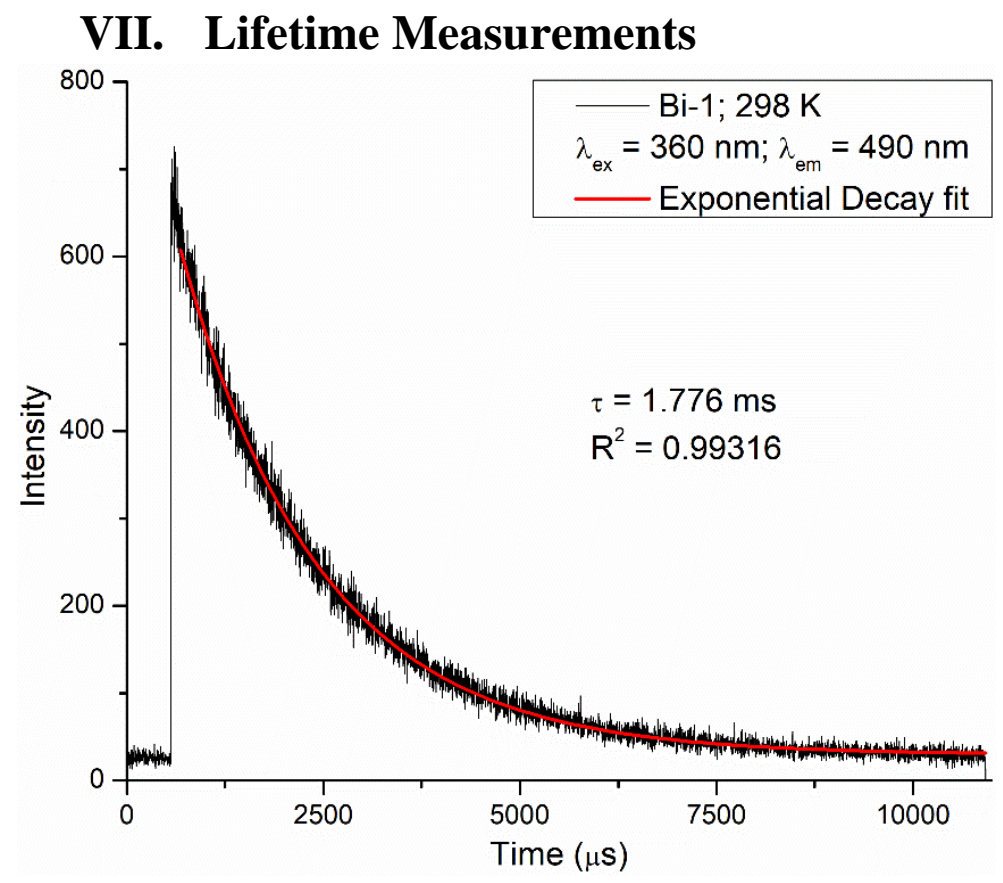

Figure S17. Phosphorescence decay plot of Bi-1 with excitation at $360 \mathrm{~nm}$ and emission at $490 \mathrm{~nm}$ recorded at $298 \mathrm{~K}$.

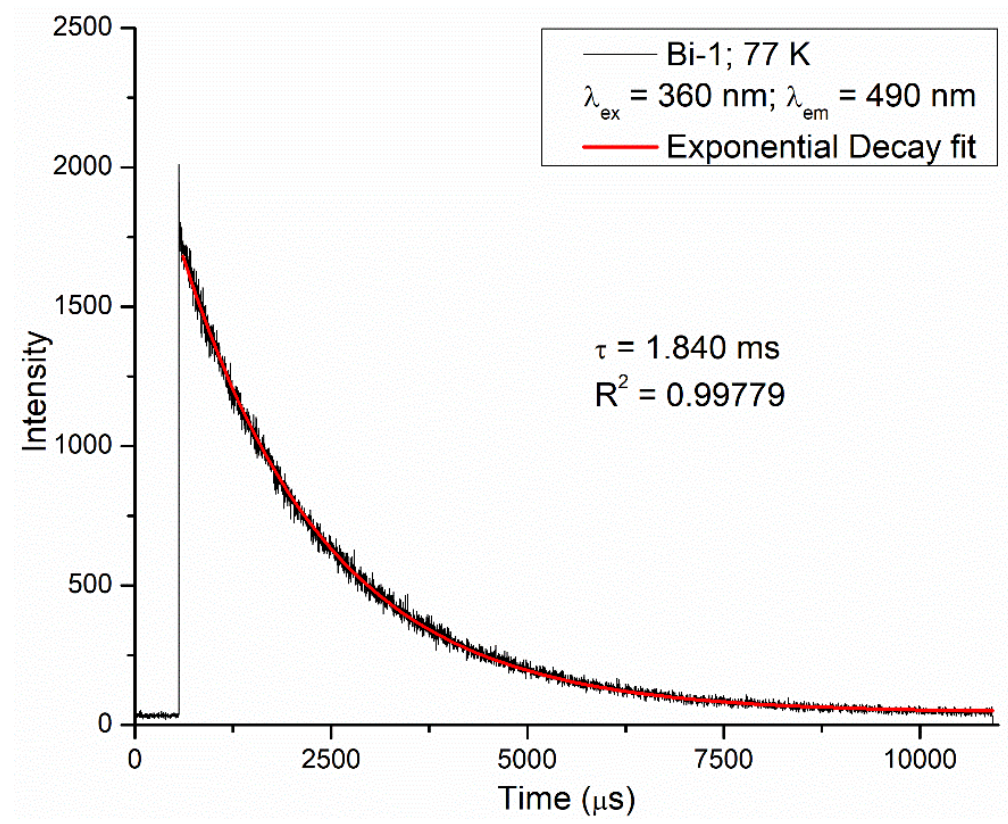

Figure S18. Phosphorescence decay plot of Bi-1 with excitation at $360 \mathrm{~nm}$ and emission at $490 \mathrm{~nm}$ recorded at $77 \mathrm{~K}$. 


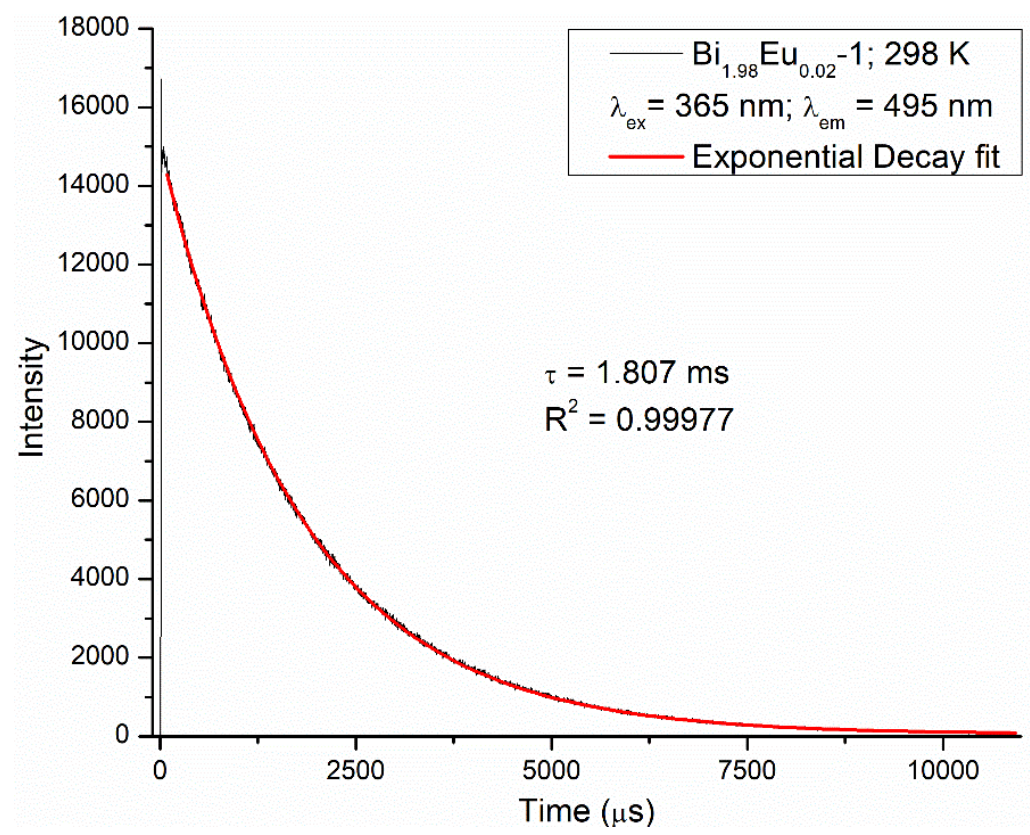

Figure S19. Phosphorescence decay plot of $\mathbf{B i}_{1.98} \mathbf{E u}_{0.02}-\mathbf{1}$ with excitation at $365 \mathrm{~nm}$ and emission at 495 $\mathrm{nm}$ recorded at $298 \mathrm{~K}$.

The decay plots shown in Figures S20 and S22-S26 were fit with an exponential decay function beginning later in the spectra $(2000+\mu \mathrm{s})$. There appears to be a simultaneous growth and decay mechanism that is more pronounced at the start of data collection, precluding an accurate exponential decay function fit for the entire data set.

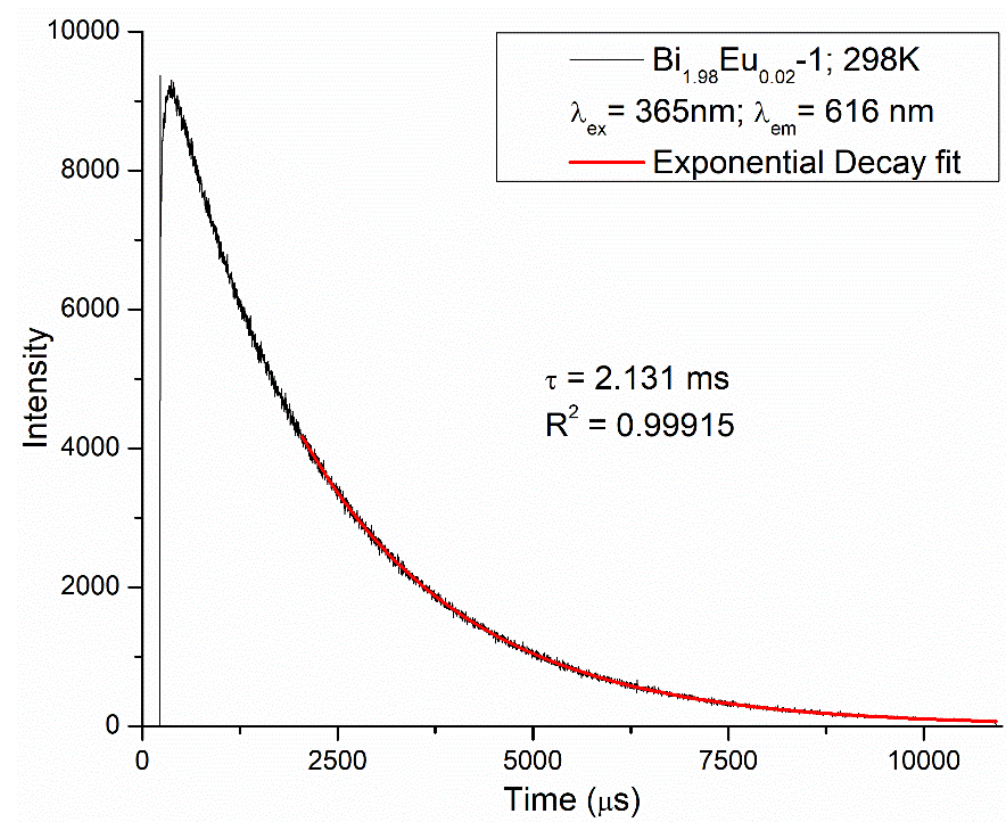

Figure S20. Phosphorescence decay plot of $\mathbf{B i}_{1.98} \mathbf{E u}_{\mathbf{0 . 0 2}}-\mathbf{1}$ with excitation at $365 \mathrm{~nm}$ and emission at 616 $\mathrm{nm}$ recorded at $298 \mathrm{~K}$. 


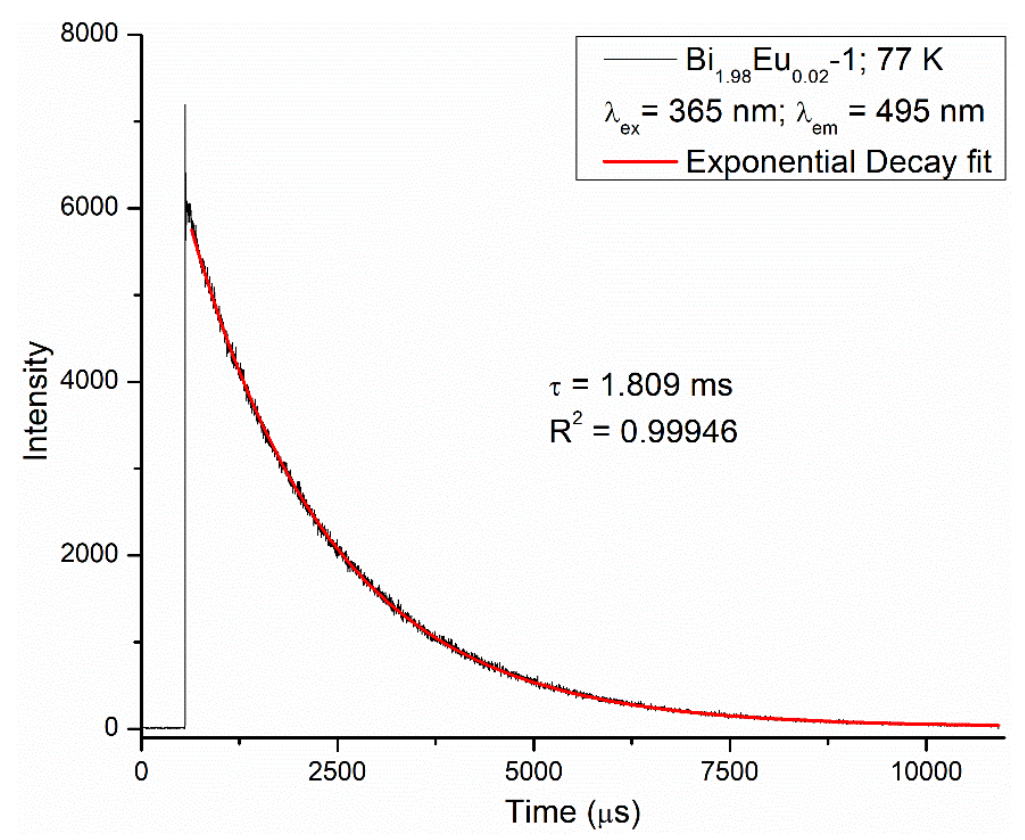

Figure S21. Phosphorescence decay plot of $\mathbf{B i}_{\mathbf{1 . 9 8}} \mathbf{E u}_{\mathbf{0 . 0 2}} \mathbf{- 1}$ with excitation at $365 \mathrm{~nm}$ and emission at 495 $\mathrm{nm}$ recorded at $77 \mathrm{~K}$.

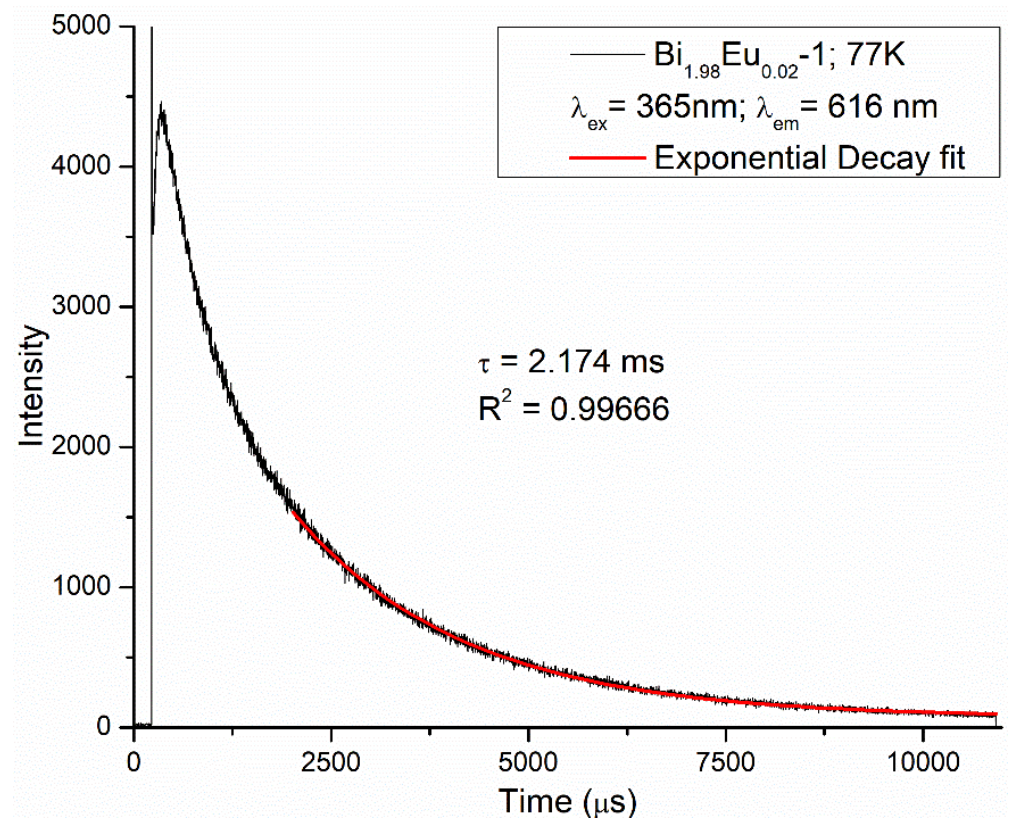

Figure S22. Phosphorescence decay plot of $\mathbf{B i}_{\mathbf{1 . 9 8}} \mathbf{E u}_{0.02}-\mathbf{1}$ with excitation at $365 \mathrm{~nm}$ and emission at 616 $\mathrm{nm}$ recorded at $77 \mathrm{~K}$. 


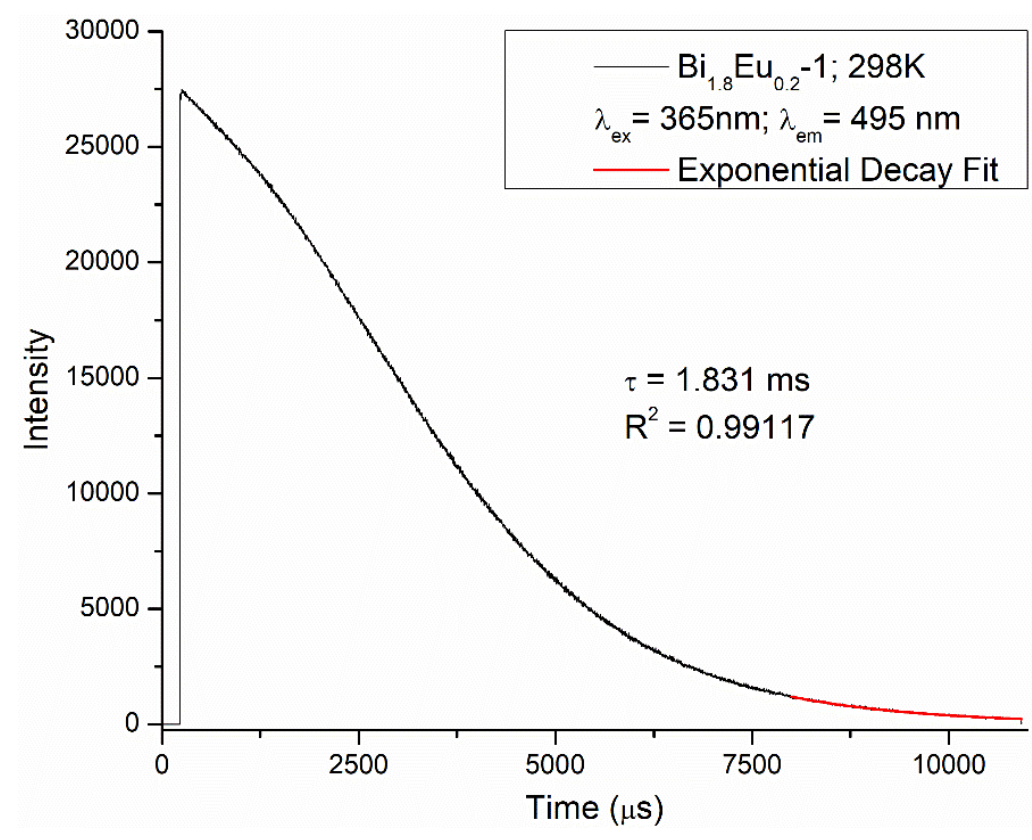

Figure S23. Phosphorescence decay plot of $\mathbf{B} \mathbf{i}_{1.8} \mathbf{E u}_{0.2}-\mathbf{1}$ with excitation at $365 \mathrm{~nm}$ and emission at $495 \mathrm{~nm}$ recorded at $298 \mathrm{~K}$.

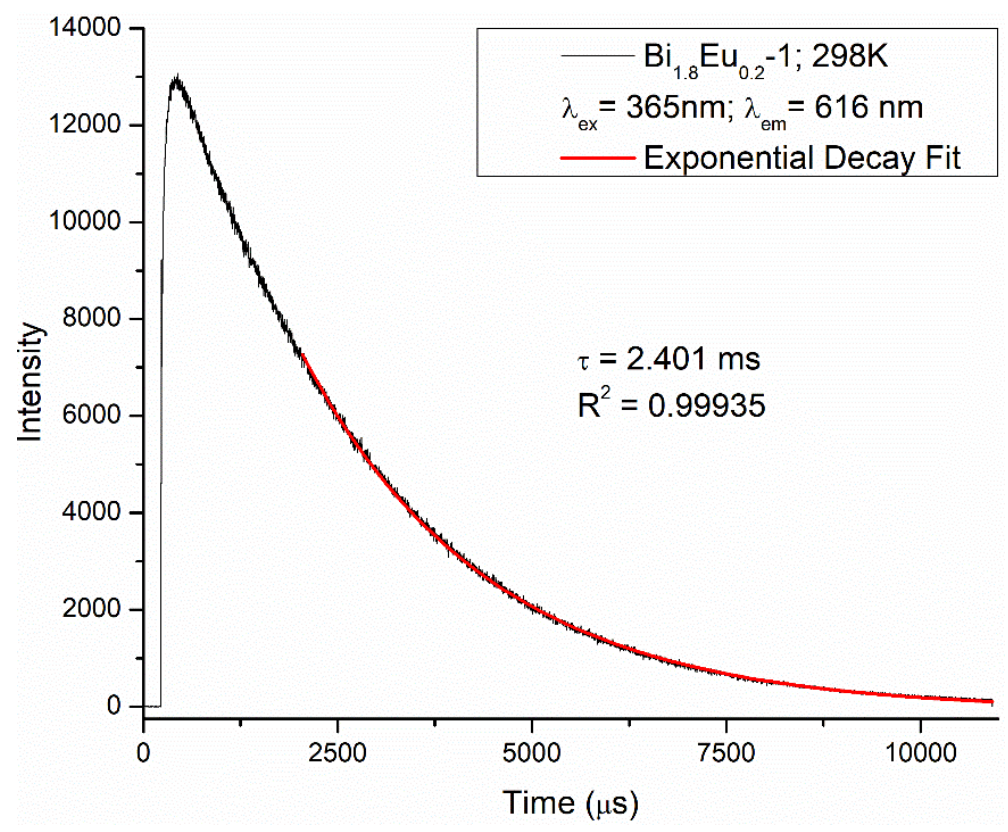

Figure S24. Phosphorescence decay plot of $\mathbf{B i}_{1.8} \mathbf{E u}_{0.2}-\mathbf{1}$ with excitation at $365 \mathrm{~nm}$ and emission at $616 \mathrm{~nm}$ recorded at $298 \mathrm{~K}$. 


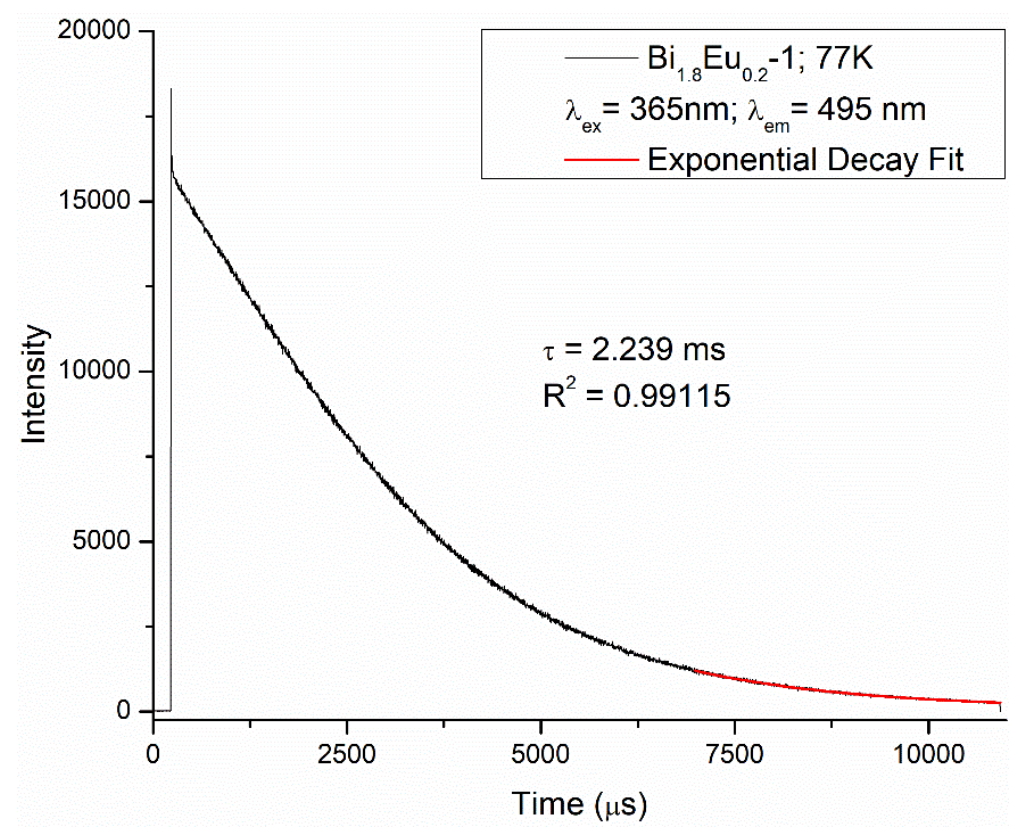

Figure S25. Phosphorescence decay plot of $\mathbf{B} \mathbf{i}_{1.8} \mathbf{E u}_{0.2}-\mathbf{1}$ with excitation at $365 \mathrm{~nm}$ and emission at $495 \mathrm{~nm}$ recorded at $77 \mathrm{~K}$.

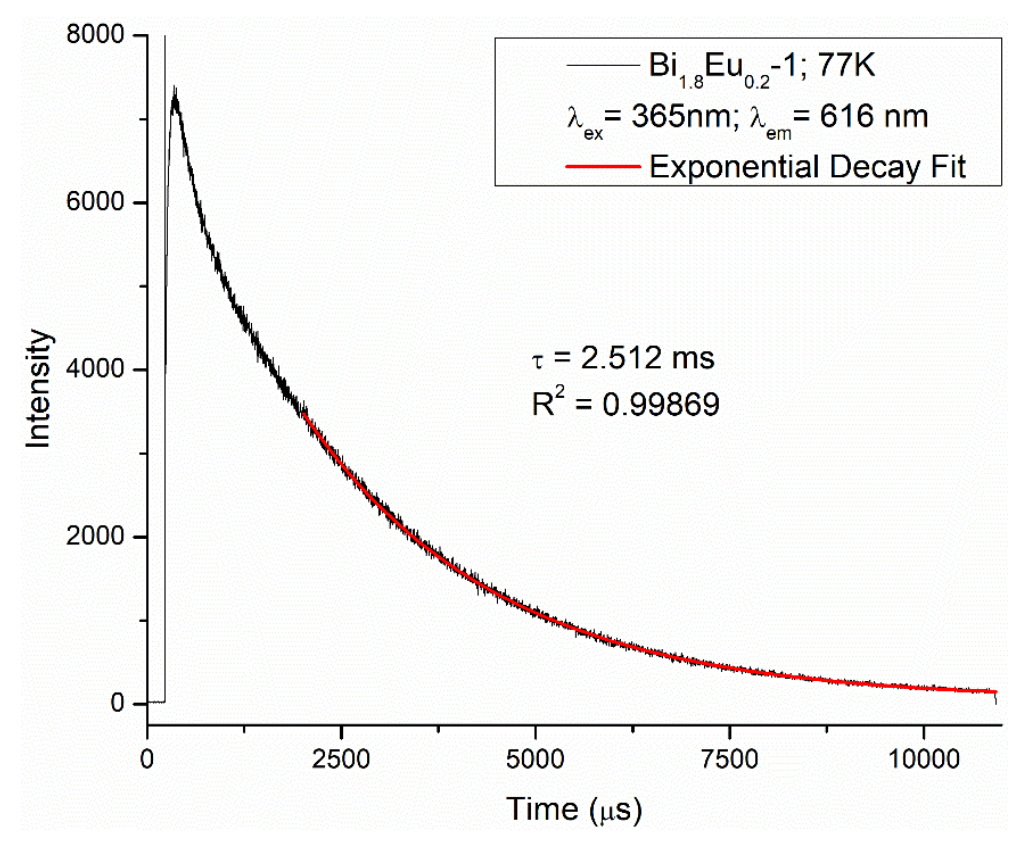

Figure S26. Phosphorescence decay plot of $\mathbf{B i}_{1.8} \mathbf{E u}_{0.2}-\mathbf{1}$ with excitation at $365 \mathrm{~nm}$ and emission at $616 \mathrm{~nm}$ recorded at $77 \mathrm{~K}$. 


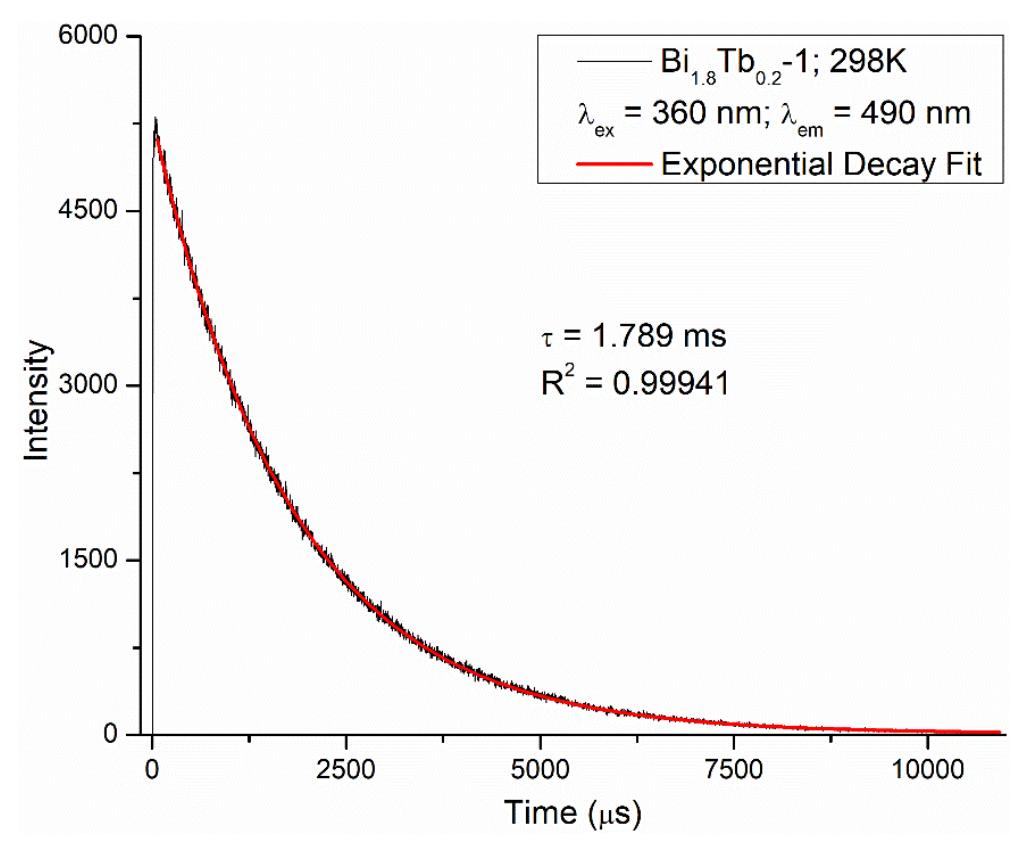

Figure S27. Phosphorescence decay plot of $\mathbf{B i}_{1.8} \mathbf{T b}_{\mathbf{0 . 2}} \mathbf{- 1}$ with excitation at $360 \mathrm{~nm}$ and emission at $490 \mathrm{~nm}$ recorded at $298 \mathrm{~K}$.

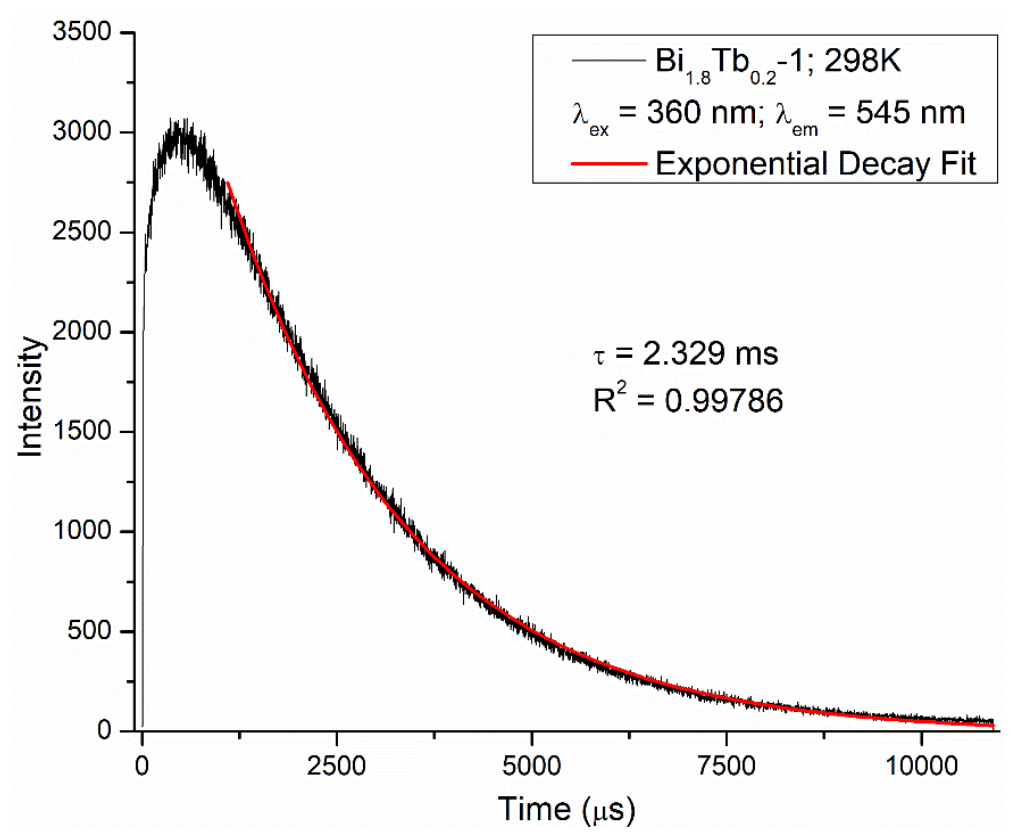

Figure S28. Phosphorescence decay plot of $\mathbf{B i}_{1.8} \mathbf{T b}_{\mathbf{0 . 2}} \mathbf{- 1}$ with excitation at $360 \mathrm{~nm}$ and emission at $545 \mathrm{~nm}$ recorded at $298 \mathrm{~K}$. 


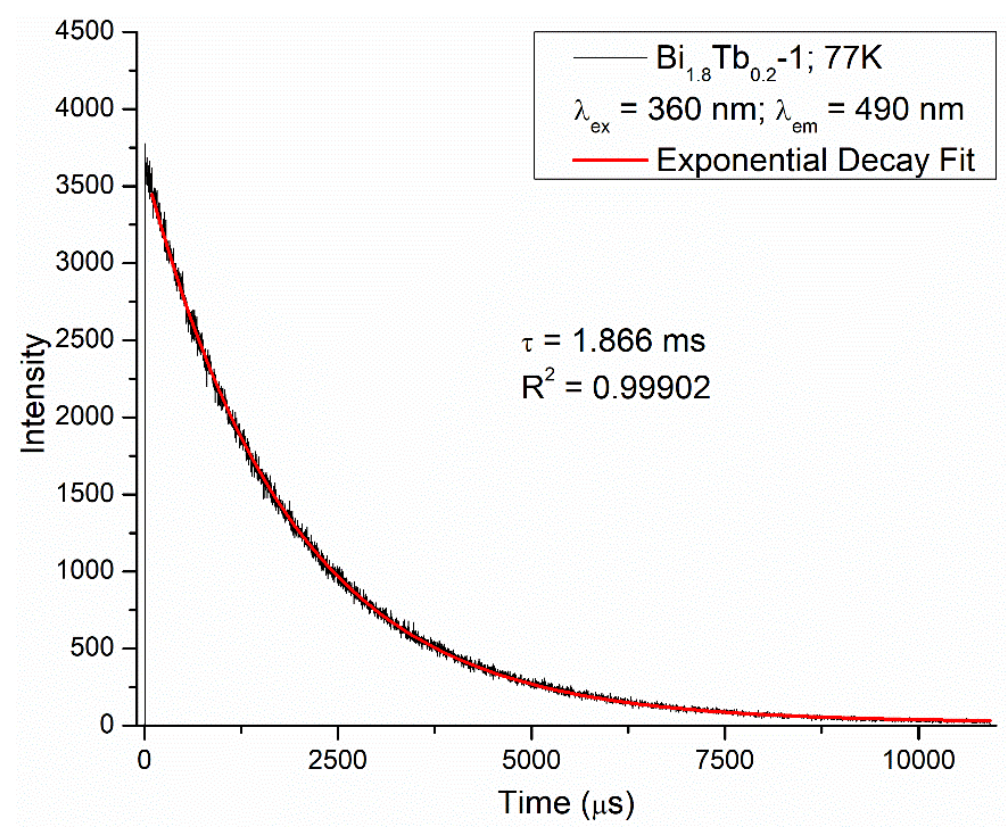

Figure S29. Phosphorescence decay plot of $\mathbf{B i}_{1.8} \mathbf{T b}_{\mathbf{0 . 2}} \mathbf{- 1}$ with excitation at $360 \mathrm{~nm}$ and emission at $490 \mathrm{~nm}$ recorded at $77 \mathrm{~K}$.

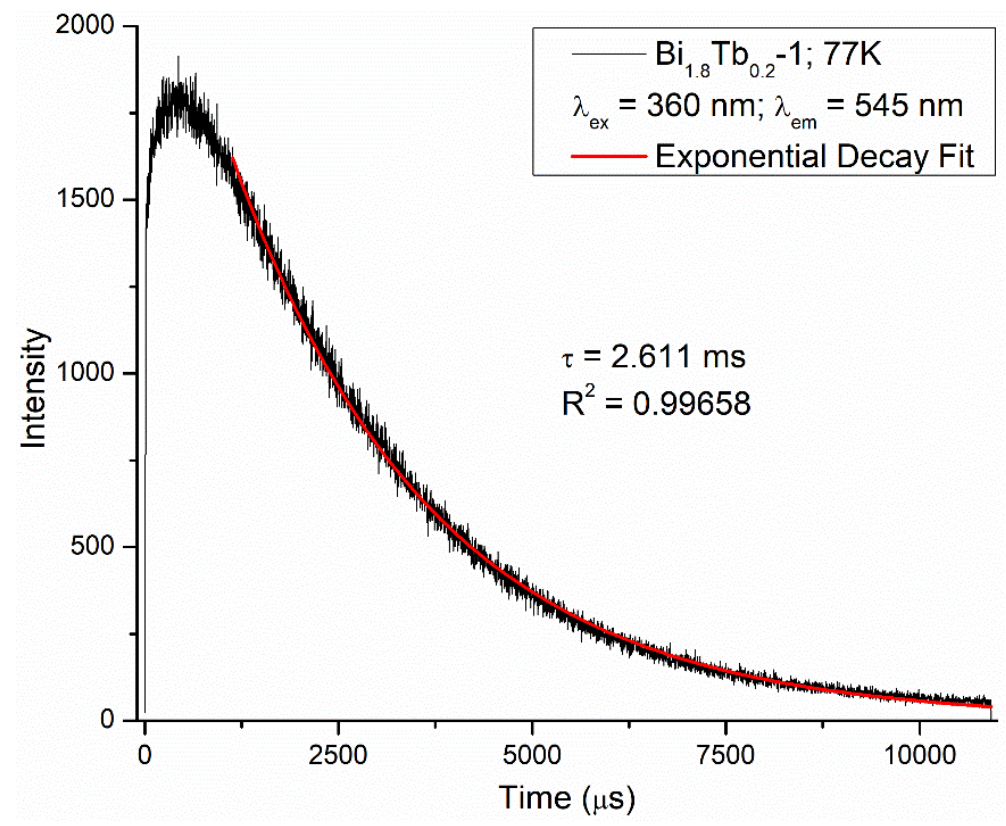

Figure S30. Phosphorescence decay plot of $\mathbf{B i}_{1.8} \mathbf{T b}_{\mathbf{0 . 2}} \mathbf{- 1}$ with excitation at $360 \mathrm{~nm}$ and emission at $545 \mathrm{~nm}$ recorded at $77 \mathrm{~K}$. 


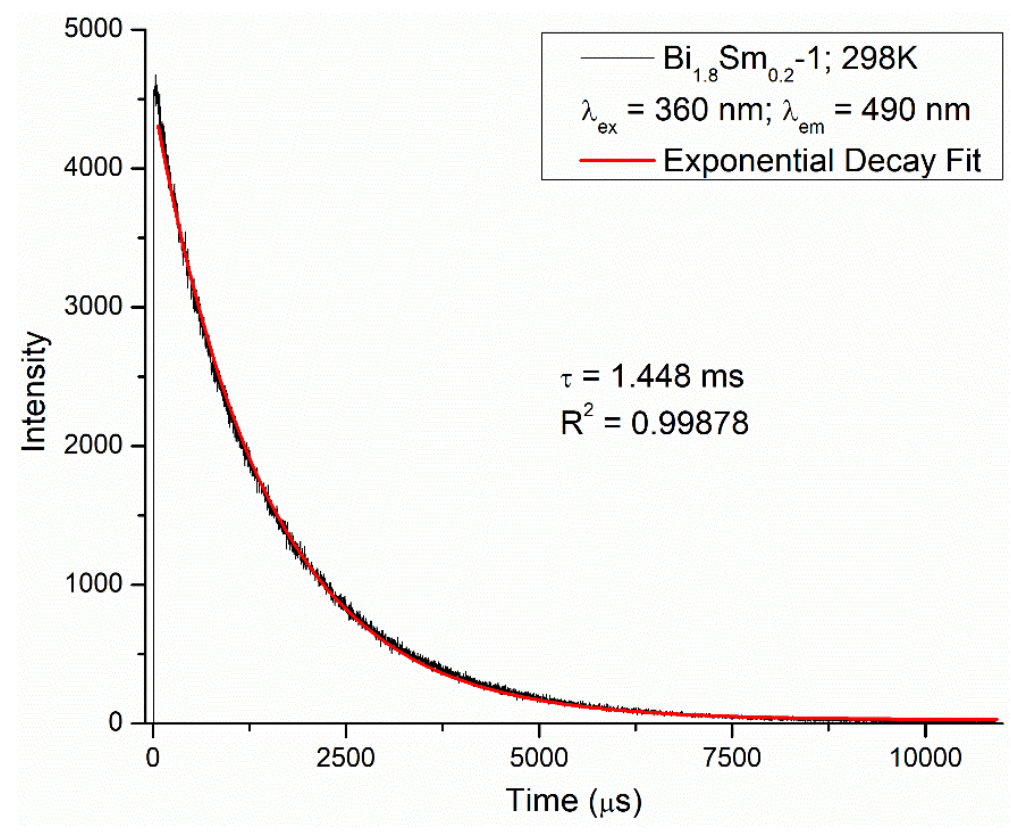

Figure S31. Phosphorescence decay plot of $\mathbf{B i}_{1.8} \mathbf{S m}_{0.2} \mathbf{- 1}$ with excitation at $360 \mathrm{~nm}$ and emission at $490 \mathrm{~nm}$ recorded at $298 \mathrm{~K}$.

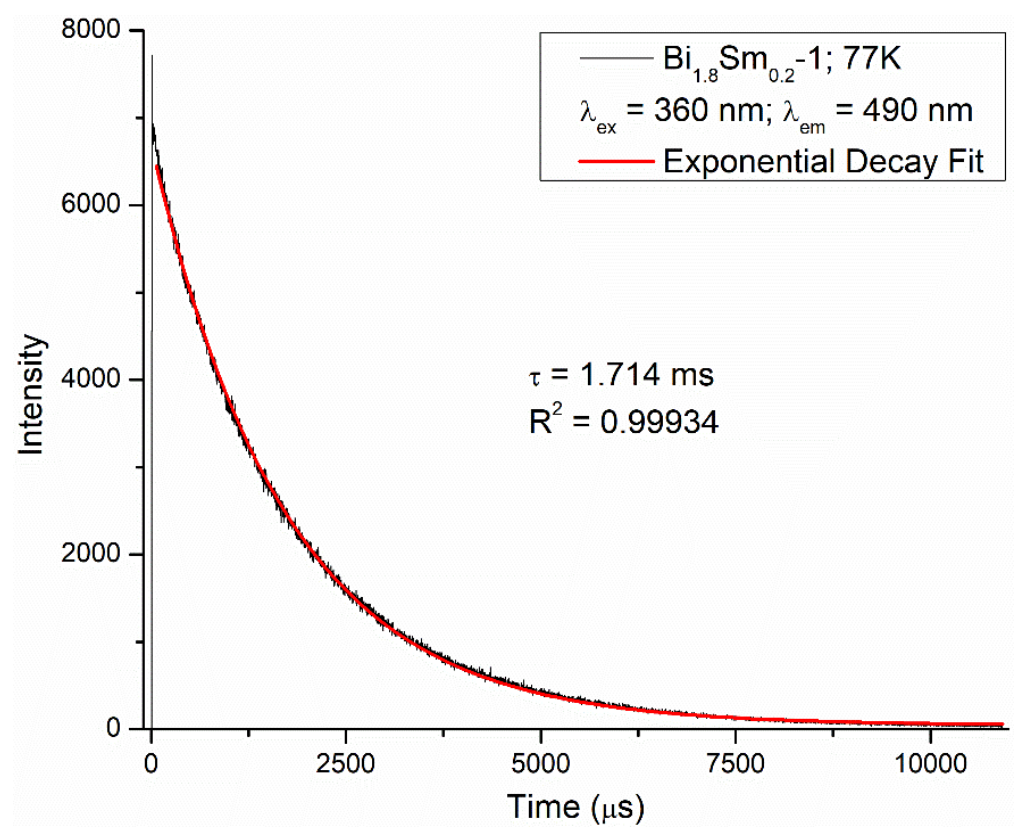

Figure S32. Phosphorescence decay plot of $\mathbf{B i}_{1.8} \mathbf{S m}_{0.2} \mathbf{- 1}$ with excitation at $360 \mathrm{~nm}$ and emission at $490 \mathrm{~nm}$ recorded at $77 \mathrm{~K}$. 


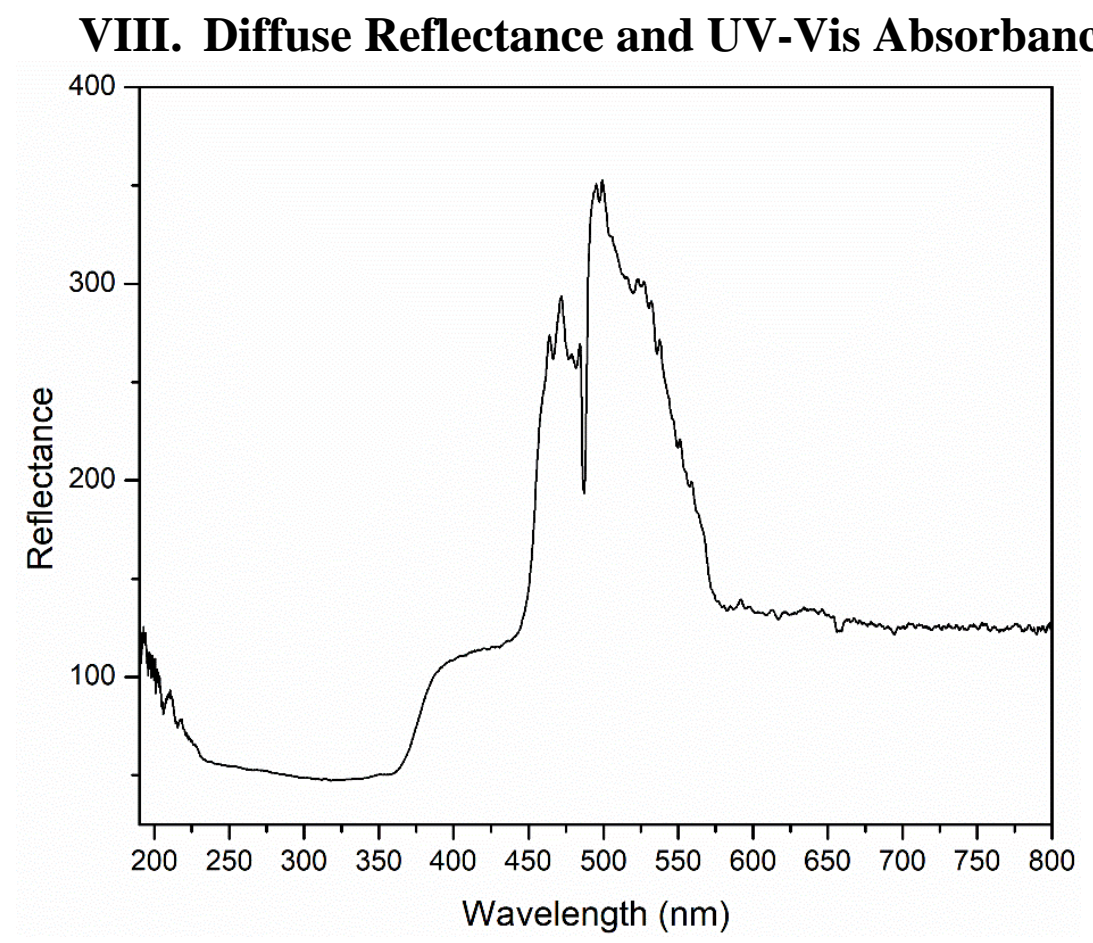

Figure S33. Diffuse reflectance spectrum of Bi-1.

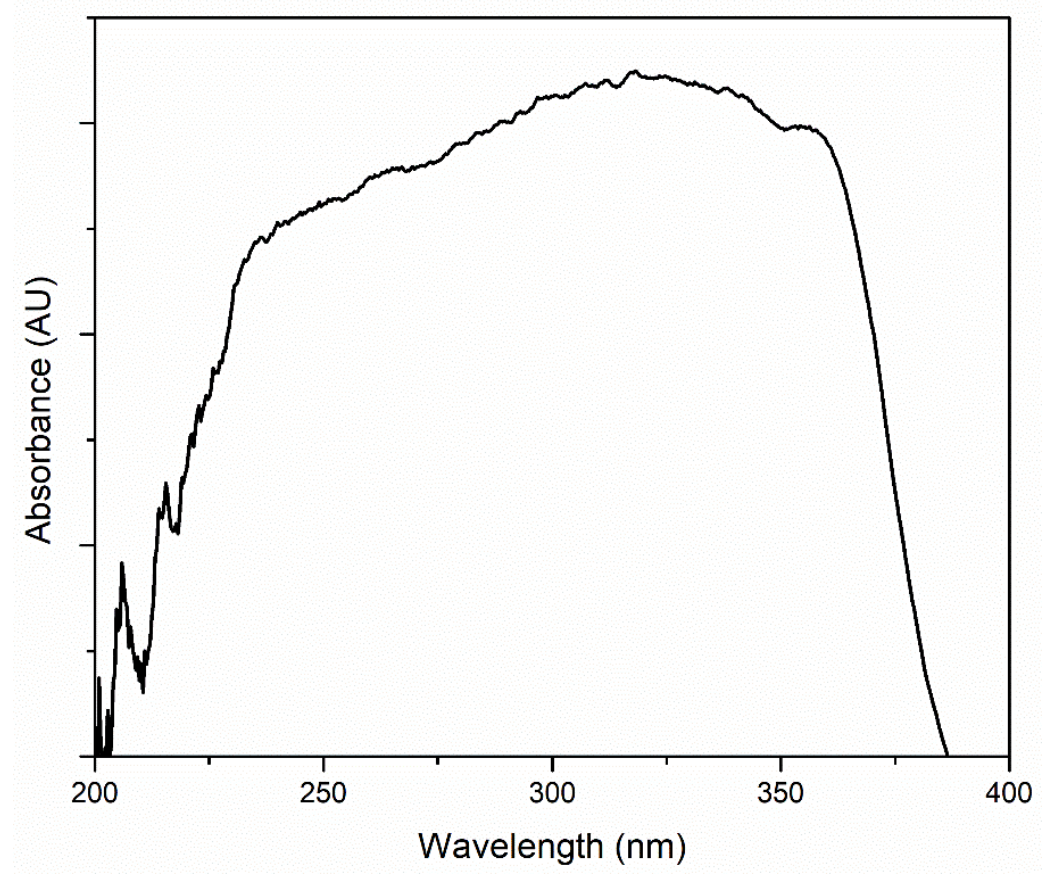

Figure S34. Absorbance of Bi-1 in the UV region, converted from diffuse reflectance data. 


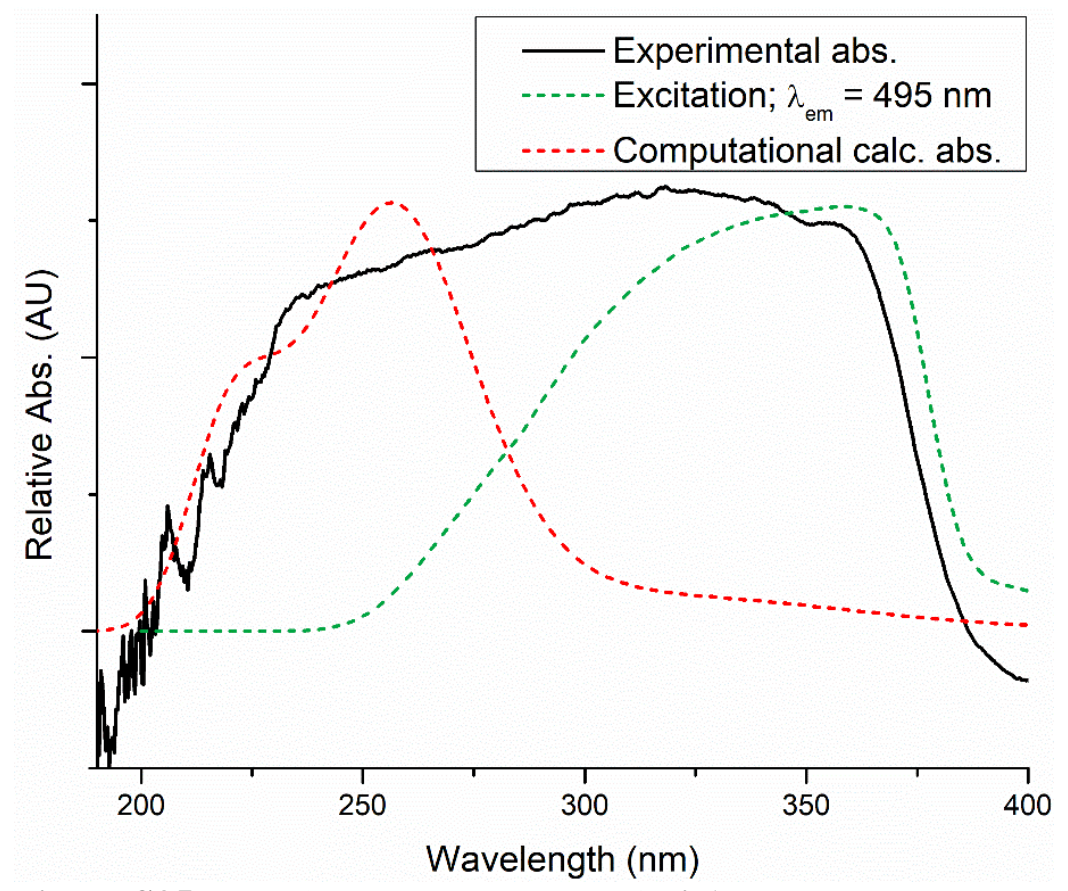

Figure S35. Experimental absorbance of Bi-1 (black) overlaid with calculated absorbance spectrum from TD-DFT (red) and excitation spectrum from emission at $495 \mathrm{~nm}$ (green). 


\section{Supramolecular Interactions}

Noncovalent interactions including $\pi-\pi$ stacking, metal- $\pi$ interactions, and hydrogen bonding were calculated using the PLATON software suite via the "Calc All" feature. These supramolecular interactions are shown in the table below, with literature precedence for their classification as such. ${ }^{1-5}$

Table S1. Significant supramolecular interactions $(\pi-\pi$ stacking, metal- $\pi$ interactions, and hydrogen bonding) observed in Bi-1. $\mathrm{C}_{\mathrm{g} 1}=$ centroid formed by $\mathrm{N}_{11}, \mathrm{C}_{12}, \mathrm{C}_{13}, \mathrm{C}_{14}, \mathrm{C}_{15}, \mathrm{C}_{16} . \mathrm{C}_{\mathrm{g} 3}=$ centroid formed by $\mathrm{N}_{31}, \mathrm{C}_{31}, \mathrm{C}_{32}, \mathrm{C}_{33}, \mathrm{C}_{34}, \mathrm{C}_{35}$. $\mathrm{C}_{\mathrm{g} 4}=$ centroid formed by $\mathrm{N}_{32}, \mathrm{C}_{38}, \mathrm{C}_{39}, \mathrm{C}_{40}, \mathrm{C}_{41}, \mathrm{C}_{42}$. $\mathrm{C}_{\mathrm{g} 5}=$ centroid formed by $\mathrm{C}_{34}, \mathrm{C}_{35}, \mathrm{C}_{36}, \mathrm{C}_{37}, \mathrm{C}_{38}, \mathrm{C}_{39}$.

\begin{tabular}{|c|c|c|c|c|}
\hline Interaction & $\begin{array}{c}\text { Distance }(\AA), \\
\mathrm{C}_{\mathrm{g}}---\mathrm{C}_{\mathrm{g}} \text { or } \mathrm{M}---\mathrm{C}_{\mathrm{g}}\end{array}$ & $\beta\left(^{\circ}\right)$ & $\begin{array}{c}\text { Distance }(\AA), \\
\text { D-H---A }\end{array}$ & $\begin{array}{c}\text { Angle }\left(^{\circ}\right), \\
\text { D-H---A }\end{array}$ \\
\hline $\mathrm{C}_{\mathrm{g} 1^{---}} \mathrm{C}_{\mathrm{g} 3}$ & $3.470(4)$ & 20.4 & - & - \\
\hline $\mathrm{C}_{\mathrm{g} 1}{ }^{---} \mathrm{C}_{\mathrm{g} 4}$ & $3.507(4)$ & 16.3 & - & - \\
\hline $\mathrm{C}_{\mathrm{g} 1---\mathrm{C}_{\mathrm{g} 5}}$ & $3.827(4)$ & 32.8 & - & - \\
\hline $\mathrm{C}_{\mathrm{g} 1---\mathrm{C}_{\mathrm{g} 5}}$ & $3.881(5)$ & 29.7 & - & - \\
\hline $\mathrm{Bi}(1)---\mathrm{C}_{\mathrm{g} 5} *$ & 3.919 & 28.20 & - & - \\
\hline $\mathrm{O}(24)-\mathrm{H}---\mathrm{O}(51)$ & - & - & $2.475(9)$ & 151(4) \\
\hline $\mathrm{O}(24)-\mathrm{H}---\mathrm{O}(51 \mathrm{~B})$ & - & - & $2.512(8)$ & $166(5)$ \\
\hline N(31)-H---O(42) & - & - & $2.707(12)$ & 150 \\
\hline $\begin{array}{c}\mathrm{N}(31)-\mathrm{H}---\mathrm{N}(22) \\
\text { Intra }\end{array}$ & - & - & $2.697(13)$ & 104 \\
\hline
\end{tabular}

*A potential interaction between the stereochemically active $6 \mathrm{~s}^{2}$ lone pair of $\mathrm{Bi}^{3+}$ and the centroid ring (lp$-\pi)$. 


\section{Computational Details}

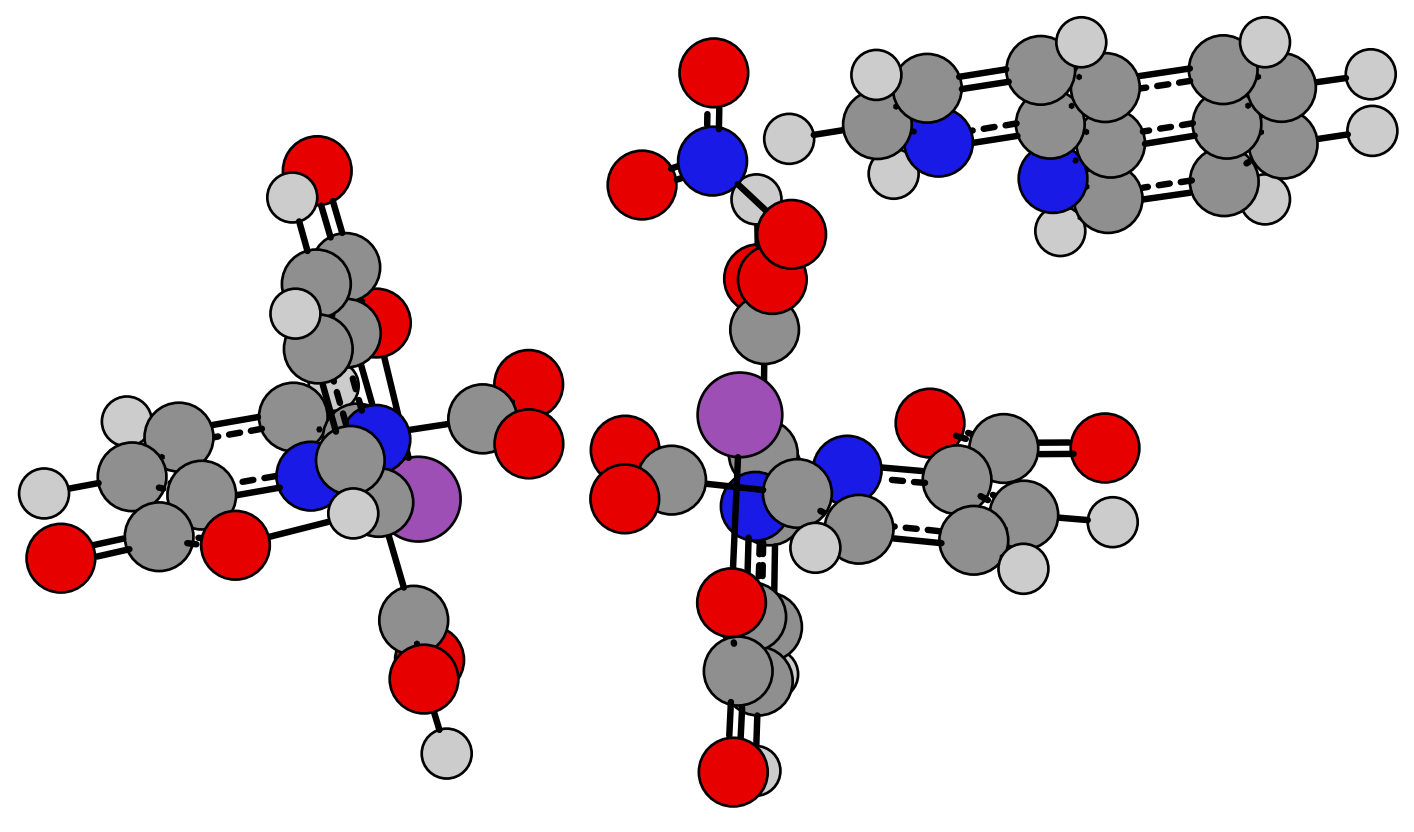

Figure S36. B3LYP optimized ground state structure of neutral model of Bi-1. 
Table S2. Selected B3LYP optimized ground state parameters of Bi-1.

\begin{tabular}{|c|c|c|c|c|c|c|c|c|c|c|}
\hline Atom1 & Atom2 & Exp & Calc & $\%$ error & Atom 1 & Atom2 & Atom3 & Exp & Calc & $\%$ error \\
\hline Bil & $\mathrm{Bi} 2$ & 4.1454 & 4.2256 & $1.9 \%$ & Bil & $\mathrm{Bi} 2$ & O74 & 162.24 & 161.45 & $0 \%$ \\
\hline Bil & O73 & 2.3277 & 2.2862 & $1.8 \%$ & Bil & $\mathrm{Bi} 2$ & O77 & 34.65 & 33.61 & $3 \%$ \\
\hline Bil & O77 & 2.5301 & 2.499 & $1.2 \%$ & Bil & $\mathrm{Bi} 2$ & O78 & 34.04 & 36.4 & $7 \%$ \\
\hline Bil & O78 & 2.4908 & 2.6536 & $6.5 \%$ & Bil & $\mathrm{Bi} 2$ & O82 & 75.89 & 73.07 & $4 \%$ \\
\hline Bil & O81 & 2.2004 & 2.1793 & $1.0 \%$ & Bil & $\mathrm{Bi} 2$ & O86 & 123.43 & 121.5 & $2 \%$ \\
\hline Bil & O85 & 2.6949 & 2.8837 & $7.0 \%$ & Bil & $\mathrm{Bi} 2$ & O89 & 92.82 & 79.51 & $14 \%$ \\
\hline $\mathrm{Bi} 2$ & O74 & 2.3277 & 2.3315 & $0.2 \%$ & Bi1 & $\mathrm{Bi} 2$ & O90 & 77.4 & 102.77 & $33 \%$ \\
\hline $\mathrm{Bi} 2$ & O77 & 2.4908 & 2.639 & $5.9 \%$ & Bi1 & O73 & $\mathrm{C} 3$ & 123.43 & 124.38 & $1 \%$ \\
\hline $\mathrm{Bi} 2$ & O78 & 2.5301 & 2.5329 & $0.1 \%$ & Bil & O77 & $\mathrm{Bi} 2$ & 111.3 & 110.63 & $1 \%$ \\
\hline $\mathrm{Bi} 2$ & O82 & 2.2004 & 2.2628 & $2.8 \%$ & Bi1 & O77 & C15 & 121.32 & 122.32 & $1 \%$ \\
\hline $\mathrm{Bi} 2$ & O86 & 2.6949 & 2.8952 & $7.4 \%$ & Bi1 & O78 & $\mathrm{Bi} 2$ & 111.3 & 109.1 & $2 \%$ \\
\hline $\mathrm{Bi} 2$ & O89 & 3.0745 & 3.0323 & $1.4 \%$ & Bi1 & O78 & C16 & 126.26 & 124.91 & $1 \%$ \\
\hline \multirow[t]{9}{*}{$\mathrm{Bi} 2$} & O90 & 3.0563 & 2.7156 & $11.1 \%$ & Bil & O81 & $\mathrm{C} 17$ & 123.79 & 125.97 & $2 \%$ \\
\hline & & & & & Bil & O85 & C29 & 116.71 & 113.74 & $3 \%$ \\
\hline & & & & & $\mathrm{Bi} 2$ & Bi1 & O85 & 123.43 & 111.02 & $10 \%$ \\
\hline & & & & & $\mathrm{Bi} 2$ & O74 & $\mathrm{C} 4$ & 123.43 & 123.72 & $0 \%$ \\
\hline & & & & & $\mathrm{Bi} 2$ & O77 & $\mathrm{C} 15$ & 126.26 & 126.41 & $0 \%$ \\
\hline & & & & & $\mathrm{Bi} 2$ & O78 & $\mathrm{C} 16$ & 121.32 & 121.16 & $0 \%$ \\
\hline & & & & & $\mathrm{Bi} 2$ & O82 & $\mathrm{C} 18$ & 123.79 & 127.28 & $3 \%$ \\
\hline & & & & & $\mathrm{Bi} 2$ & O86 & C30 & 116.7 & 114.55 & $2 \%$ \\
\hline & & & & & $\mathrm{Bi} 2$ & O89 & N72 & 98.63 & 92.46 & $6 \%$ \\
\hline
\end{tabular}




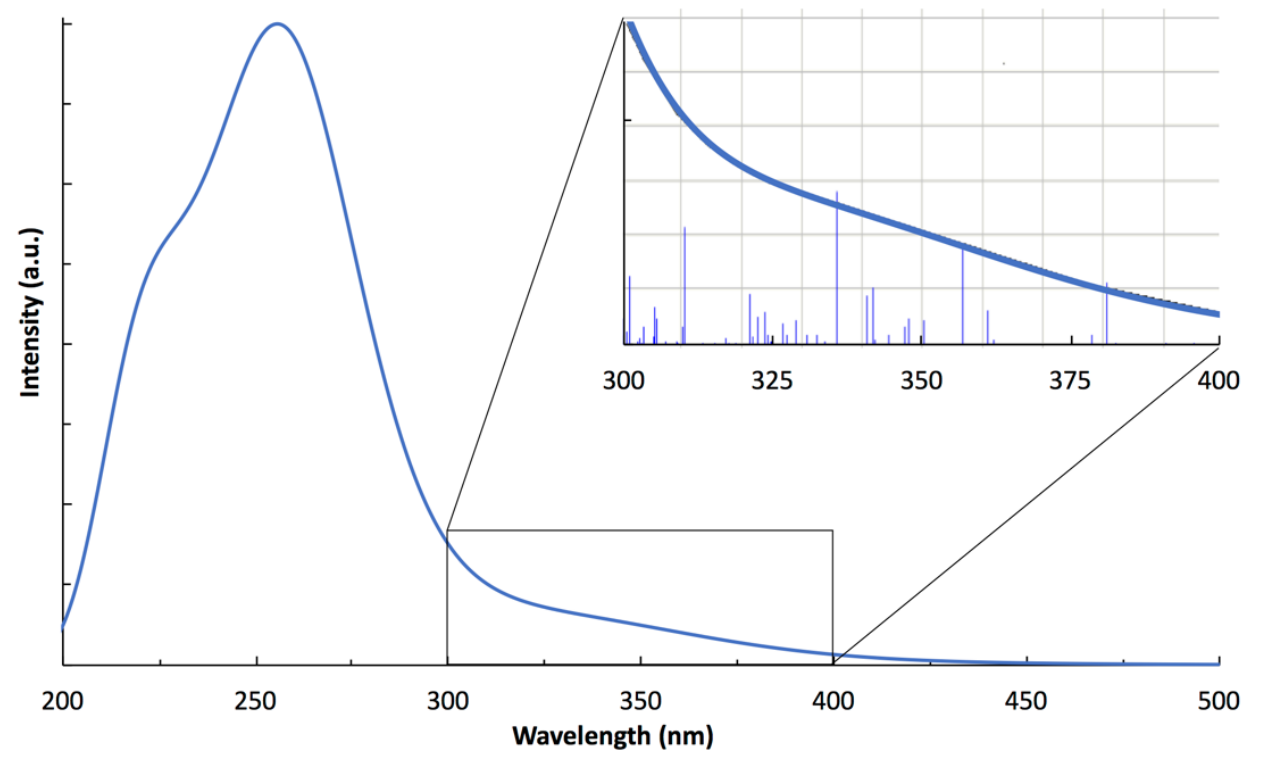

Figure S37. TD-DFT B3LYP calculated UV-vis for Bi-1. A peak FWHM of $0.333 \mathrm{eV}$ was used to convolute the spectrum. 
Table S3. TD-DFT B3LYP calculated excited singlet states for Bi-1.

\begin{tabular}{ccc}
\hline State & Wavelength & f-Oscillation \\
\hline Excited State 1 & $461.75 \mathrm{~nm}$ & 0.0001 \\
Excited State 2 & $452.92 \mathrm{~nm}$ & 0.0010 \\
Excited State 3 & $434.70 \mathrm{~nm}$ & 0.0001 \\
Excited State 4 & $423.87 \mathrm{~nm}$ & 0.0000 \\
Excited State 5 & $414.58 \mathrm{~nm}$ & 0.0000 \\
Excited State 6 & $395.11 \mathrm{~nm}$ & 0.0000 \\
Excited State 7 & $390.37 \mathrm{~nm}$ & 0.0000 \\
Excited State 8 & $382.13 \mathrm{~nm}$ & 0.0000 \\
Excited State 9 & $380.66 \mathrm{~nm}$ & 0.0044 \\
Excited State 10 & $378.11 \mathrm{~nm}$ & 0.0005 \\
Excited State 11 & $361.92 \mathrm{~nm}$ & 0.0002 \\
Excited State 12 & $360.95 \mathrm{~nm}$ & 0.0024 \\
Excited State 13 & $356.66 \mathrm{~nm}$ & 0.0072 \\
Excited State 14 & $350.19 \mathrm{~nm}$ & 0.0016 \\
Excited State 15 & $347.77 \mathrm{~nm}$ & 0.0018 \\
Excited State 16 & $347.04 \mathrm{~nm}$ & 0.0011 \\
Excited State 17 & $344.52 \mathrm{~nm}$ & 0.0005 \\
Excited State 18 & $342.13 \mathrm{~nm}$ & 0.0002 \\
Excited State 19 & $341.77 \mathrm{~nm}$ & 0.0040 \\
Excited State 20 & $340.87 \mathrm{~nm}$ & 0.0034 \\
Excited State 21 & $335.89 \mathrm{~nm}$ & 0.0111 \\
Excited State 22 & $333.81 \mathrm{~nm}$ & 0.0001 \\
Excited State 23 & $332.54 \mathrm{~nm}$ & 0.0005 \\
Excited State 24 & $330.90 \mathrm{~nm}$ & 0.0005 \\
Excited State 25 & $329.02 \mathrm{~nm}$ & 0.0016 \\
Excited State 26 & $327.50 \mathrm{~nm}$ & 0.0005 \\
Excited State 27 & $326.80 \mathrm{~nm}$ & 0.0014 \\
Excited State 28 & $325.08 \mathrm{~nm}$ & 0.0001 \\
Excited State 29 & $324.83 \mathrm{~nm}$ & 0.0001 \\
\hline
\end{tabular}


Table S4. TD-DFT B3LYP calculated electronic transitions of Bi-1 for the singlet excited state at $335.89 \mathrm{~nm}$.

\begin{tabular}{ccccc}
\hline Donor & & Acceptor & Coefficient & Percent Contribution \\
\hline $238($ HOMO-17) & $\rightarrow$ & $256($ LUMO) & 0.45224 & $42 \%$ \\
$241($ HOMO-14) & $\rightarrow$ & $256($ LUMO) & -0.32521 & $22 \%$ \\
$240($ HOMO-15) & $\rightarrow$ & $256($ LUMO) & -0.21598 & $10 \%$ \\
$239($ HOMO-16) & $\rightarrow$ & $256($ LUMO) & -0.19405 & $8 \%$ \\
$244($ HOMO-11) & $\rightarrow$ & $256($ LUMO) & 0.17277 & $6 \%$ \\
$243($ HOMO-12) & $\rightarrow$ & $256($ LUMO) & 0.16033 & $5 \%$ \\
$242($ HOMO-13) & $\rightarrow$ & $256($ LUMO) & 0.14783 & $5 \%$ \\
$231($ HOMO-24) & $\rightarrow$ & $257($ LUMO 1$)$ & -0.10529 & $2 \%$ \\
\hline
\end{tabular}




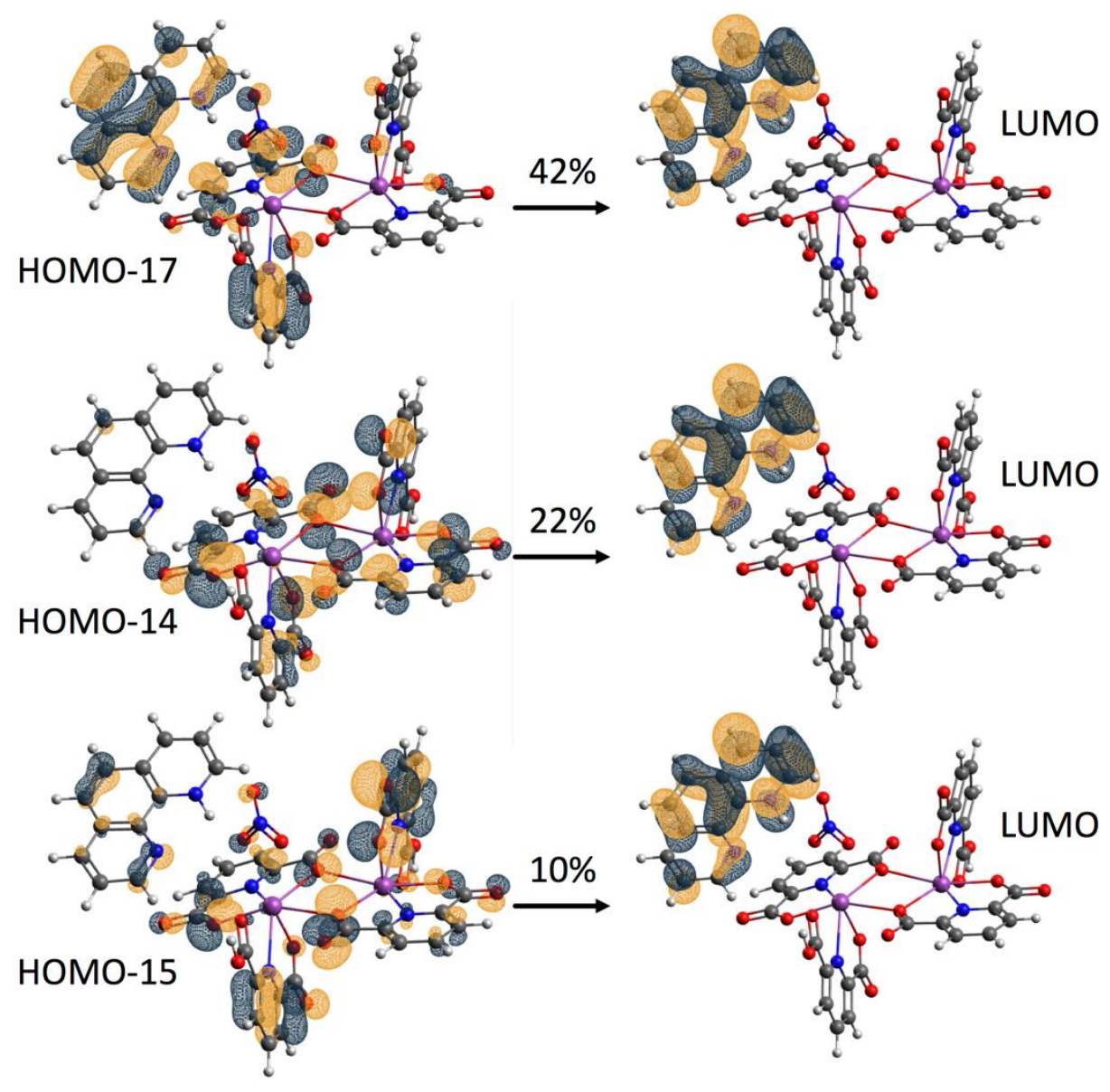

Figure S38. Isodensity representation of dominant molecular orbitals involved in TD-DFT B3LYP calculated transition at $335.89 \mathrm{~nm}$ for Bi-1. 


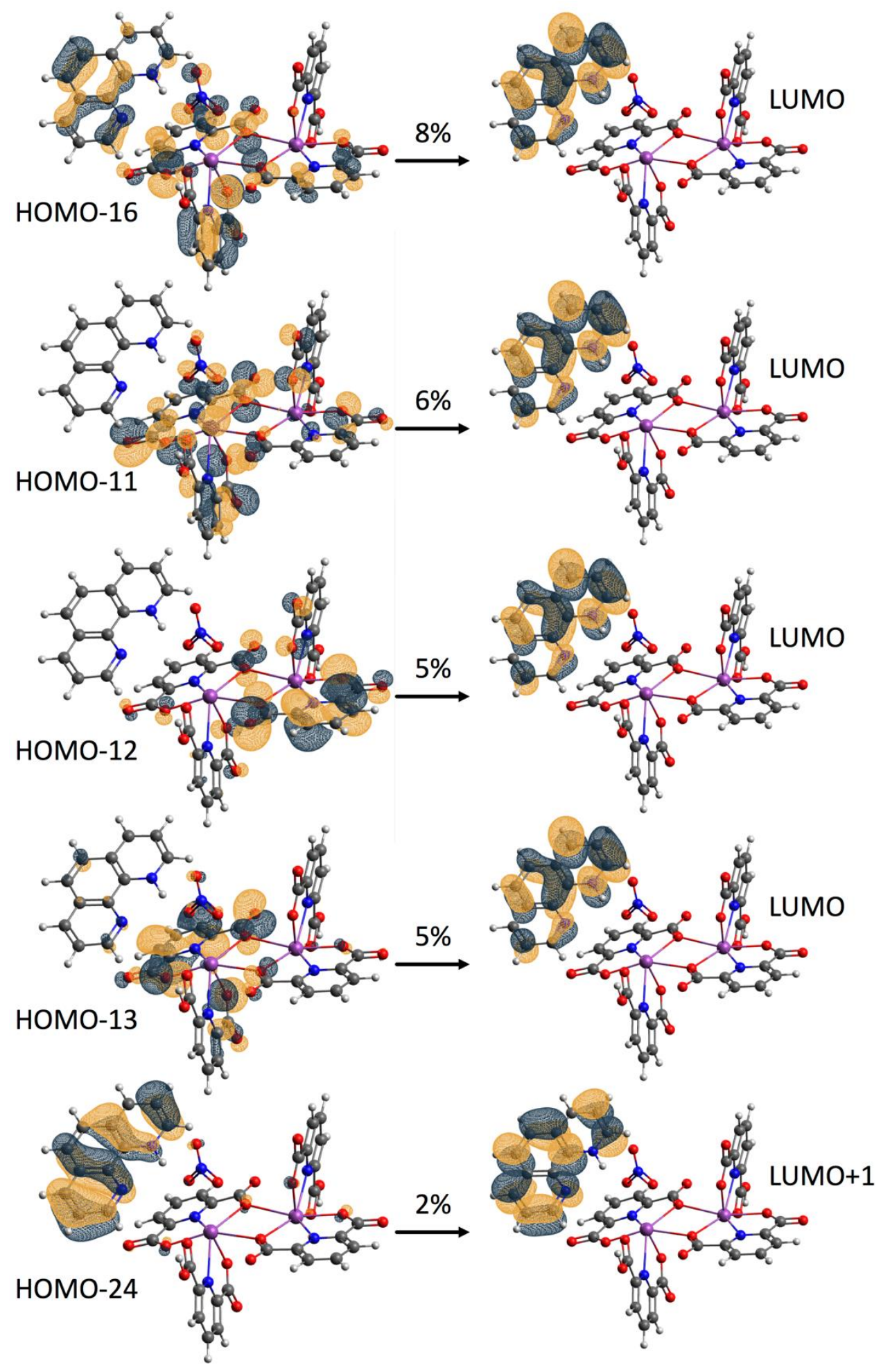

Figure S39. Isodensity representation of negligible molecular orbitals involved in TD-DFT B3LYP calculated transition at $335.89 \mathrm{~nm}$ for Bi-1. 
Table S5. TD-DFT B3LYP calculated electronic transitions of Bi-1 for the singlet excited state at $360.95 \mathrm{~nm}$.

\begin{tabular}{ccccc}
\hline Donor & & Acceptor & Coefficient & Percent Contribution \\
\hline $252($ HOMO-3) & $\rightarrow$ & $257(\mathrm{LUMO}+1)$ & 0.53895 & $62 \%$ \\
$251(\mathrm{HOMO}-4)$ & $\rightarrow$ & $257(\mathrm{LUMO}+1)$ & -0.33228 & $24 \%$ \\
$252(\mathrm{HOMO}-3)$ & $\rightarrow$ & $257(\mathrm{LUMO}+1)$ & 0.25791 & $14 \%$ \\
\hline
\end{tabular}



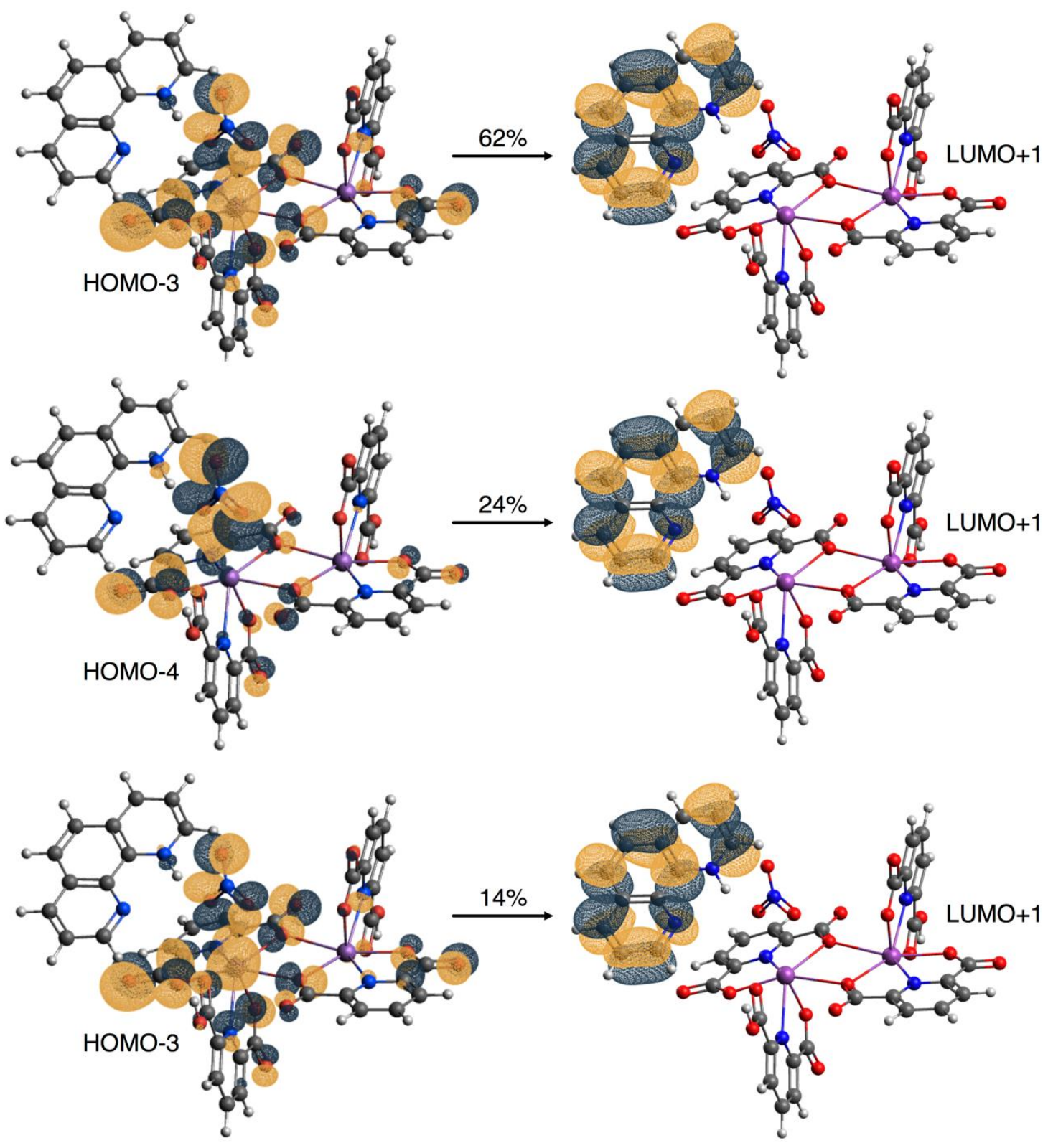

Figure S40. Isodensity representation of molecular orbitals involved in TD-DFT B3LYP calculated transition at $360.95 \mathrm{~nm}$ for $\mathbf{B i}-\mathbf{1}$. 
Table S6. TD-DFT B3LYP calculated electronic transitions of Bi-1 for the singlet excited state at $341.77 \mathrm{~nm}$

\begin{tabular}{ccccc}
\hline Donor & & Acceptor & Coefficient & Percent Contribution \\
\hline $242($ HOMO-13) & $\rightarrow$ & $256($ LUMO) & 0.61149 & $82 \%$ \\
$239($ HOMO-16) & $\rightarrow$ & $256($ LUMO) & 0.19535 & $8 \%$ \\
$245($ HOMO-10) & $\rightarrow$ & $256($ LUMO) & -0.14300 & $4 \%$ \\
$236($ HOMO-19) & $\rightarrow$ & $256($ LUMO) & -0.11717 & $3 \%$ \\
$238($ HOMO-17) & $\rightarrow$ & $256($ LUMO) & -0.10956 & $3 \%$ \\
\hline
\end{tabular}




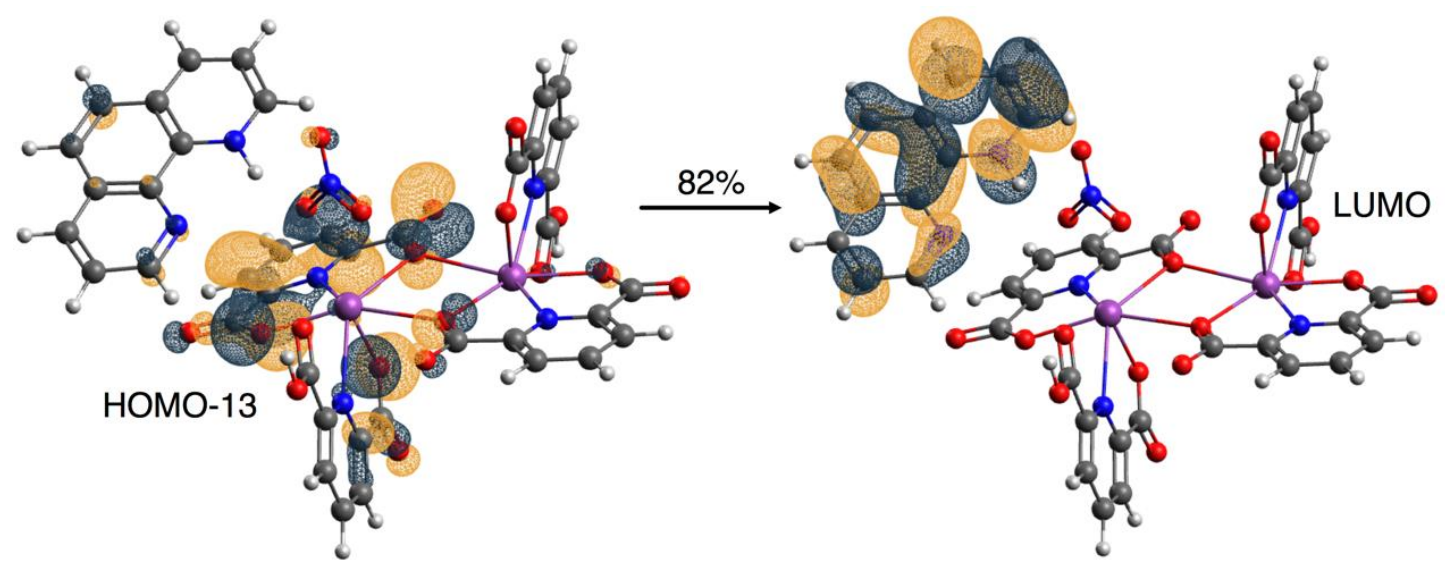

Figure S41. Isodensity representation of the molecular orbitals involved in TD-DFT B3LYP calculated transition at $341.77 \mathrm{~nm}$ for $\mathbf{B i}-\mathbf{1}$ for contributions $>10 \%$. 
Table S7. TD-DFT B3LYP calculated electronic transitions of Bi-1 for the singlet excited state at $340.87 \mathrm{~nm}$.

\begin{tabular}{ccccc}
\hline Donor & & Acceptor & Coefficient & Percent Contribution \\
\hline $255(\mathrm{HOMO})$ & $\rightarrow$ & $258(\mathrm{LUMO}+2)$ & 0.65585 & $91 \%$ \\
$254(\mathrm{HOMO}-1)$ & $\rightarrow$ & $258(\mathrm{LUMO}+2)$ & 0.18463 & $7 \%$ \\
$250(\mathrm{HOMO}-5)$ & $\rightarrow$ & $258(\mathrm{LUMO}+2)$ & 0.10192 & $2 \%$ \\
\hline
\end{tabular}




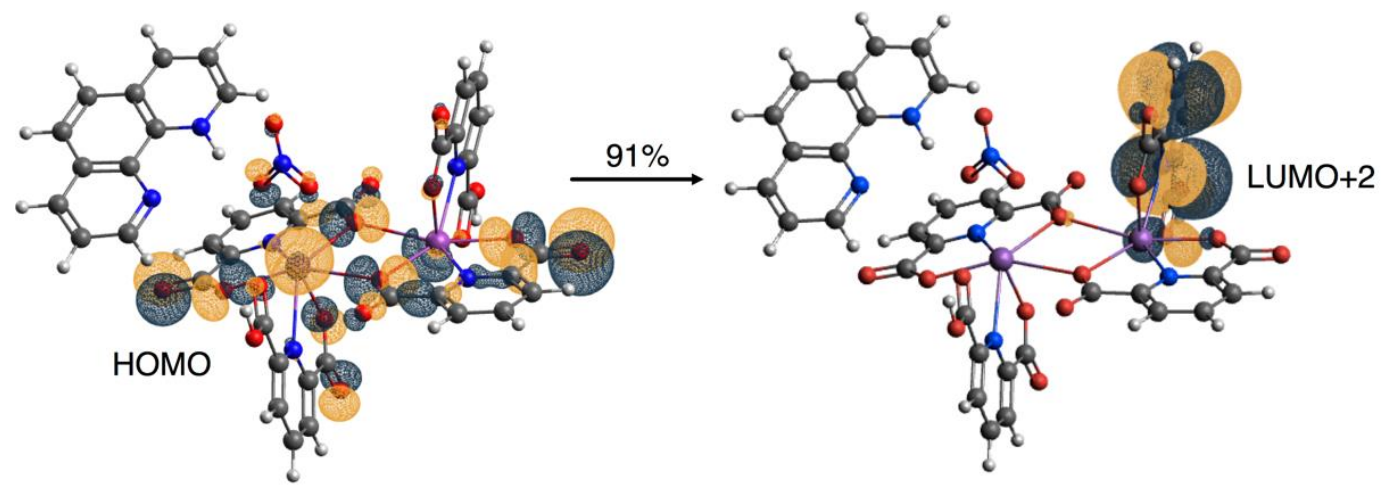

Figure S42. Isodensity representation of molecular orbitals involved in TD-DFT B3LYP calculated transition at $340.87 \mathrm{~nm}$ for $\mathbf{B i}-\mathbf{1}$ for contributions $>10 \%$. 


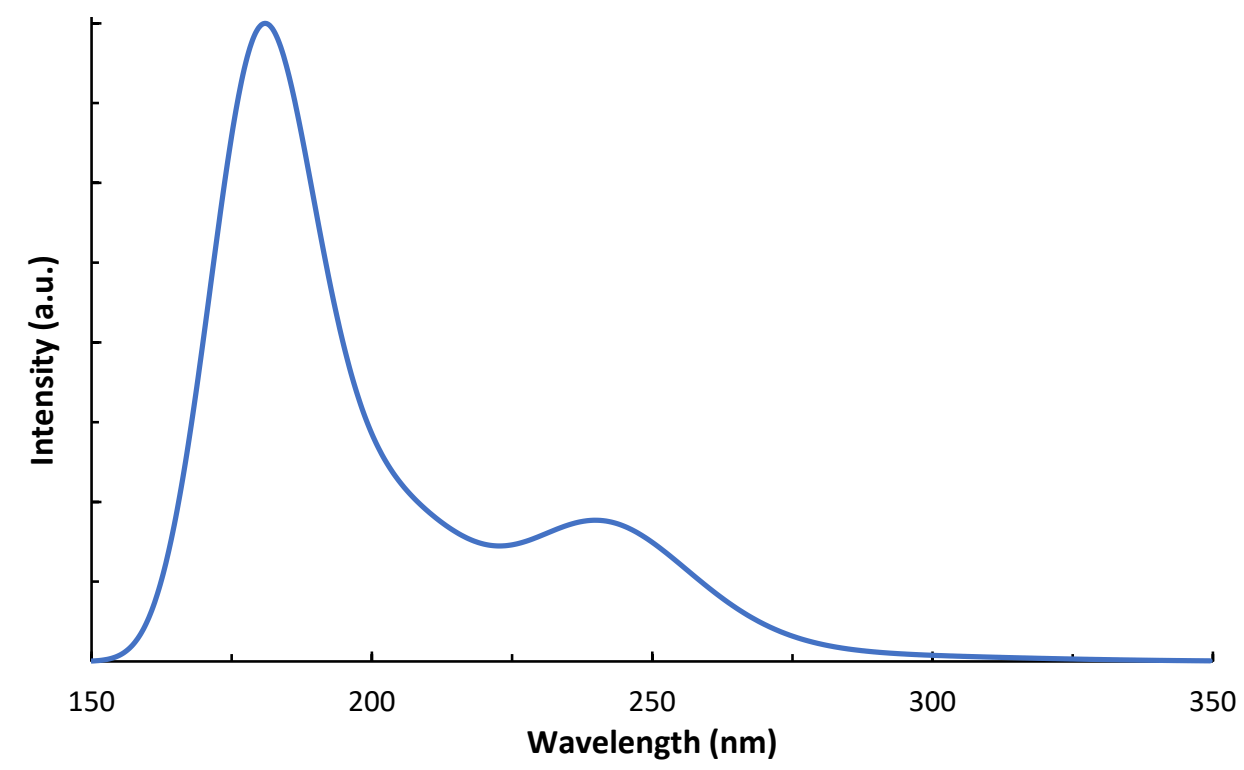

Figure S43. TD-DFT CAM-B3LYP calculated UV-vis for Bi-1. A peak FWHM of $0.333 \mathrm{eV}$ was used to convolute the spectrum.

Table S8. TD-DFT CAM-B3LYP calculated excited singlet states for Bi-1.

\begin{tabular}{ccc}
\hline State & Wavelength & f-Oscillation \\
\hline Excited State 1 & $305.76 \mathrm{~nm}$ & 0.0182 \\
Excited State 2 & $293.70 \mathrm{~nm}$ & 0.0017 \\
Excited State 3 & $287.07 \mathrm{~nm}$ & 0.0098 \\
Excited State 4 & $284.06 \mathrm{~nm}$ & 0.0037 \\
Excited State 5 & $283.33 \mathrm{~nm}$ & 0.0003 \\
Excited State 6 & $281.78 \mathrm{~nm}$ & 0.0036 \\
Excited State 7 & $280.64 \mathrm{~nm}$ & 0.0002 \\
Excited State 8 & $279.71 \mathrm{~nm}$ & 0.0005 \\
Excited State 9 & $279.40 \mathrm{~nm}$ & 0.0003 \\
Excited State 10 & $277.09 \mathrm{~nm}$ & 0.0015 \\
\hline
\end{tabular}




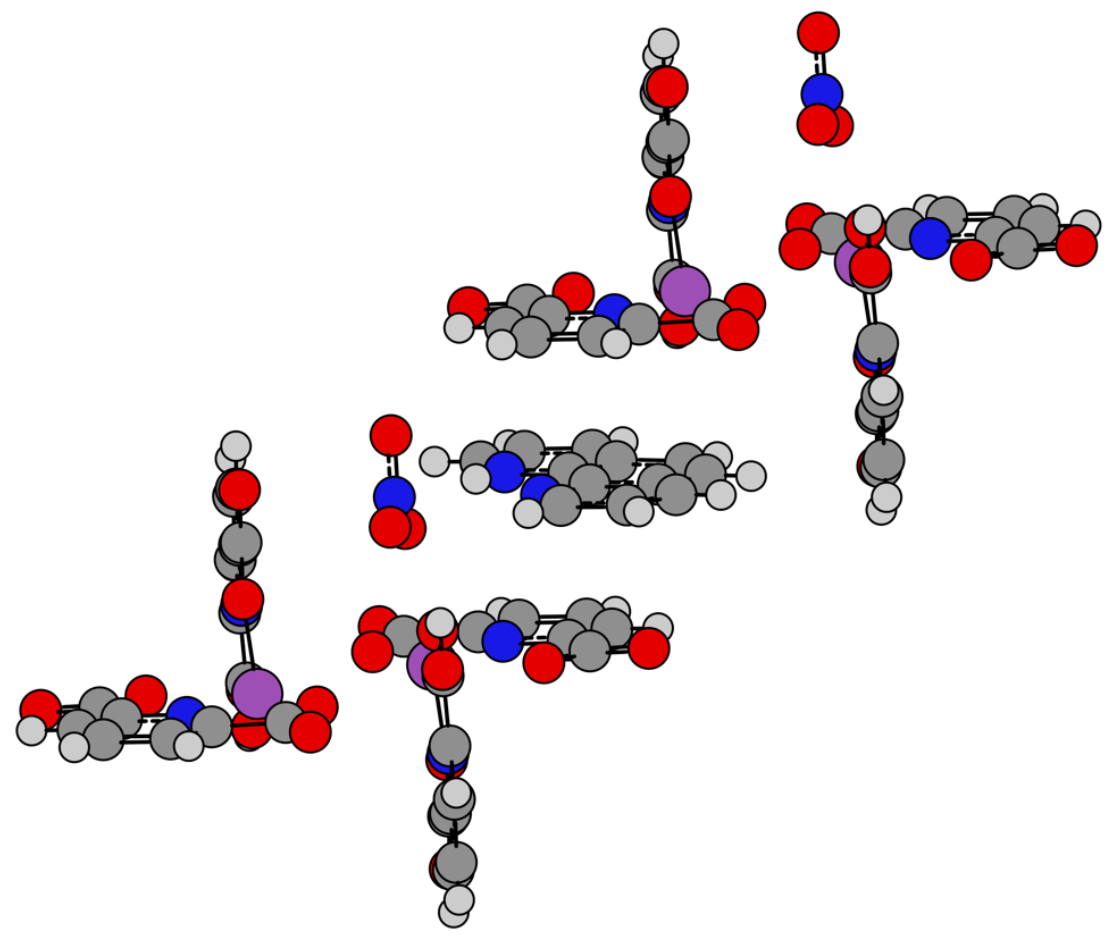

Figure S44. Crystallographic unit Bi-1, $\left[\mathrm{Bi}_{2}(\mathrm{HPDC})_{2}(\mathrm{PDC})_{2}\left(\mathrm{NO}_{3}\right) \cdot \mathrm{HPhen} \bullet \mathrm{Bi}_{2}(\mathrm{HPDC})_{2}(\mathrm{PDC})_{2}\left(\mathrm{NO}_{3}\right)\right]$, utilized for Wiberg index, second order perturbation theory, and critical bond point calculations. 

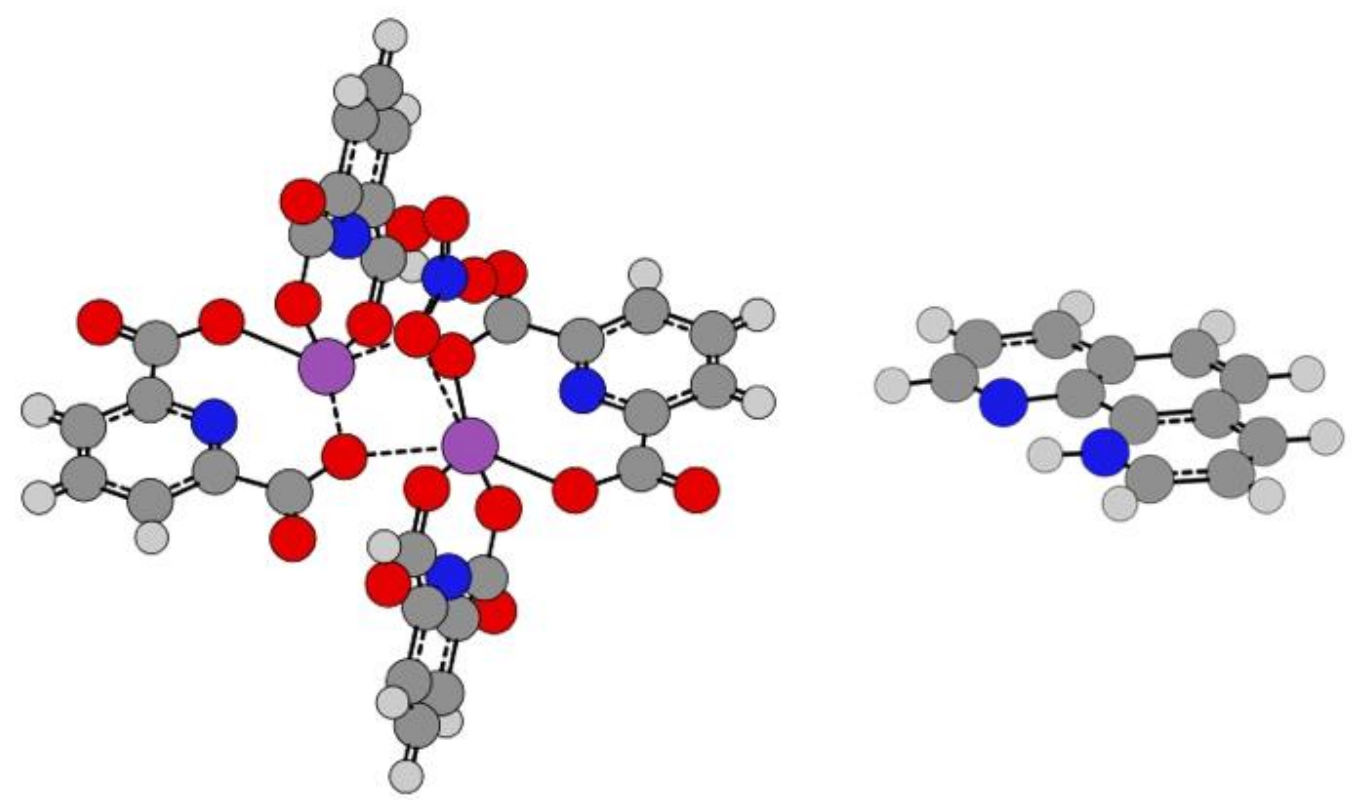

Figure S45. Crystallographic units of Bi-1, (left) $\left[\mathrm{Bi}_{2}(\mathrm{HPDC})_{2}(\mathrm{PDC})_{2}\left(\mathrm{NO}_{3}\right)\right]^{-}$and (right) $[\mathrm{HPhen}]^{+}$, utilized for electrostatic potential surfaces. 


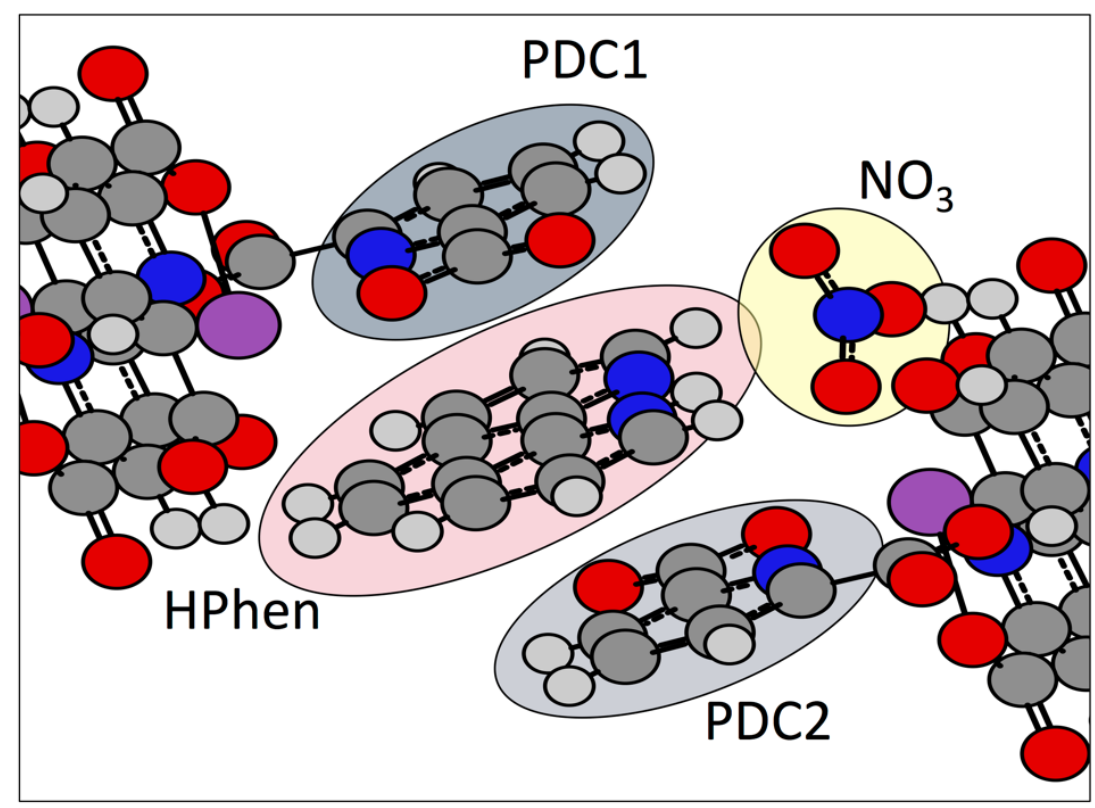

Figure S46. NCI relevant NBO calculated units of Bi-1. 
Table S9. NBO orbitals and stabilization energy in Bi-1 involved in noncovalent interactions.

\begin{tabular}{|c|c|c|c|c|c|c|c|}
\hline Donor & Acceptor & $\begin{array}{l}\text { Donor } \\
\text { NBO }\end{array}$ & Donor MOs & $\begin{array}{l}\text { Acceptor } \\
\text { NBO }\end{array}$ & Acceptor MOs & $\begin{array}{c}\text { Stabilization E } \\
(\mathrm{kJ} / \mathrm{mol})\end{array}$ & $\begin{array}{c}\text { Interaction } \\
\text { Type }\end{array}$ \\
\hline HPhen & PDC1 & 159 & LP (1) C 64 & 493 & $\mathrm{BD}^{*}(2) \mathrm{C}$ 9- C 13 & 0.46 & $\pi-\pi$ \\
\hline HPhen & PDC1 & 159 & LP (1) C 64 & 513 & BD*(2) C 17- C 21 & 0.80 & $\pi-\pi$ \\
\hline HPhen & PDC1 & 424 & $\mathrm{BD}(2) \mathrm{C} 61-\mathrm{N} 118$ & 513 & BD*(2) C 17- C 21 & 0.14 & $\pi-\pi$ \\
\hline HPhen & PDC1 & 424 & BD (2) C 61- N118 & 534 & $\mathrm{BD}^{*}(2) \mathrm{C} 25-\mathrm{N} 110$ & 0.31 & $\pi-\pi$ \\
\hline HPhen & PDC1 & 435 & BD (2) C 66- C 67 & 493 & $\mathrm{BD}^{*}(2) \mathrm{C}$ 9- C 13 & 0.10 & $\pi-\pi$ \\
\hline HPhen & PDC1 & 449 & $\mathrm{BD}(2) \mathrm{C} 72-\mathrm{N} 119$ & 477 & $\mathrm{BD}^{*}(1) \mathrm{C}$ 5- $\mathrm{O} 122$ & 0.06 & $\pi-\pi$ \\
\hline HPhen & PDC1 & 449 & BD (2) C 72- N119 & 479 & $\mathrm{BD}^{*}(2) \mathrm{C} 5-\mathrm{O} 126$ & 0.45 & $\pi-\pi$ \\
\hline HPhen & PDC1 & 449 & BD (2) C 72- N119 & 493 & $\mathrm{BD}^{*}(2) \mathrm{C}$ 9- C 13 & 0.06 & $\pi-\pi$ \\
\hline HPhen & PDC2 & 159 & LP (1) C 64 & 491 & $\mathrm{BD}^{*}(2) \mathrm{C}$ 8- $\mathrm{O} 129$ & 0.08 & $\pi-\pi$ \\
\hline HPhen & PDC2 & 168 & LP (1) N119 & 502 & BD*(2) C 12- C 16 & 0.06 & $\pi-\pi$ \\
\hline HPhen & PDC2 & 443 & $\mathrm{BD}$ (2) C 70- C 71 & 522 & $\mathrm{BD}^{*}(2)$ C $20-\mathrm{C} 24$ & 0.41 & $\pi-\pi$ \\
\hline HPhen & PDC2 & 443 & $\mathrm{BD}(2) \mathrm{C} 70-\mathrm{C} 71$ & 543 & $\mathrm{BD}^{*}(2) \mathrm{C} 28-\mathrm{N} 113$ & 0.09 & $\pi-\pi$ \\
\hline HPhen & PDC2 & 449 & BD (2) C 72- N119 & 522 & BD*(2) C 20- C 24 & 0.13 & $\pi-\pi$ \\
\hline HPhen & PDC2 & 463 & $3 \mathrm{C}(1) \mathrm{C} 65-\mathrm{C} 68-\mathrm{C} 69$ & 502 & BD*(2) C 12- C 16 & 0.12 & $\pi-\pi$ \\
\hline HPhen & PDC2 & 463 & $3 \mathrm{C}$ (1)C65-C68-C69 & 543 & $\mathrm{BD}^{*}(2) \mathrm{C} 28-\mathrm{N} 113$ & 0.07 & $\pi-\pi$ \\
\hline HPhen & $\mathrm{NO}_{3}$ & 168 & LP (1) N119 & 672 & $\mathrm{BD} *(1) \mathrm{N} 120-\mathrm{O} 158$ & 0.10 & $\mathrm{n}-\pi^{*}$ \\
\hline HPhen & $\mathrm{NO}_{3}$ & 168 & LP (1) N119 & 673 & $\mathrm{BD}^{*}(2) \mathrm{N} 120-\mathrm{O} 158$ & 0.23 & $\mathrm{n}-\pi^{*}$ \\
\hline $\mathrm{NO}_{3}$ & HPhen & 251 & LP (1) O156 & 669 & BD*(1) H109- N118 & 2.21 & HB \\
\hline $\mathrm{NO}_{3}$ & HPhen & 252 & LP (2) O156 & 669 & BD*(1) H109- N118 & 2.28 & HB \\
\hline $\mathrm{NO}_{3}$ & HPhen & 253 & LP (3) O156 & 669 & BD*(1) H109- N118 & 3.64 & HB \\
\hline $\mathrm{NO}_{3}$ & HPhen & 258 & LP (2) O158 & 669 & BD*(1) H109- N118 & 0.12 & HB \\
\hline $\mathrm{NO}_{3}$ & HPhen & 455 & BD (1) N120- O154 & 669 & BD*(1) H109- N118 & 0.09 & HB \\
\hline $\mathrm{NO}_{3}$ & HPhen & 247 & LP (3) O154 & 660 & $\mathrm{BD}^{*}$ (1) C 71- C 72 & 0.09 & $\mathrm{n}-\pi^{*}$ \\
\hline $\mathrm{NO}_{3}$ & HPhen & 251 & LP (1) O156 & 648 & $\mathrm{BD}^{*}(1) \mathrm{C} 65-\mathrm{N} 118$ & 0.13 & $\mathrm{n}-\pi^{*}$ \\
\hline $\mathrm{NO}_{3}$ & HPhen & 252 & LP (2) O156 & 648 & $\mathrm{BD}^{*}(1) \mathrm{C}$ 65- N118 & 0.11 & $\mathrm{n}-\pi^{*}$ \\
\hline $\mathrm{NO}_{3}$ & HPhen & 253 & LP (3) O156 & 648 & $\mathrm{BD}^{*}(1) \mathrm{C} 65-\mathrm{N} 118$ & 0.07 & $\mathrm{n}-\pi^{*}$ \\
\hline $\mathrm{NO}_{3}$ & HPhen & 253 & LP (3) O156 & 639 & $\mathrm{BD}^{*}(2) \mathrm{C} 61-\mathrm{N} 118$ & 0.22 & $\mathrm{n}-\pi^{*}$ \\
\hline $\mathrm{NO}_{3}$ & HPhen & 253 & LP (3) O156 & 664 & $\mathrm{BD}^{*}(2) \mathrm{C} 72-\mathrm{N} 119$ & 0.08 & $\mathrm{n}-\pi^{*}$ \\
\hline PDC1 & HPhen & 169 & LP (1) O122 & 664 & BD*(2) C 72- N119 & 0.06 & $\pi-\pi$ \\
\hline PDC1 & HPhen & 171 & LP (3) O122 & 664 & $\mathrm{BD}^{*}(2) \mathrm{C} 72-\mathrm{N} 119$ & 0.30 & $\pi-\pi$ \\
\hline PDC1 & HPhen & 182 & LP (2) O126 & 678 & $3 \mathrm{Cn}(1) \mathrm{C} 65-\mathrm{C} 68-\mathrm{C} 69$ & 0.18 & $\pi-\pi$ \\
\hline PDC1 & HPhen & 264 & $\mathrm{BD}(2) \mathrm{C} 5-\mathrm{O} 126$ & 678 & $3 \mathrm{Cn}(1) \mathrm{C} 65-\mathrm{C} 68-\mathrm{C} 69$ & 0.19 & $\pi-\pi$ \\
\hline PDC1 & HPhen & 278 & $\mathrm{BD}(2) \mathrm{C}$ 9- C 13 & 678 & $3 \mathrm{Cn}(1) \mathrm{C} 65-\mathrm{C} 68-\mathrm{C} 69$ & 0.78 & $\pi-\pi$ \\
\hline PDC1 & HPhen & 298 & $\mathrm{BD}$ (2) C 17- C 21 & 679 & $3 C^{*}(1) \mathrm{C} 65-\mathrm{C} 68-\mathrm{C} 69$ & 0.06 & $\pi-\pi$ \\
\hline PDC1 & HPhen & 298 & $\mathrm{BD}$ (2) C 17- C 21 & 678 & $3 \mathrm{Cn}(1) \mathrm{C} 65-\mathrm{C} 68-\mathrm{C} 69$ & 0.24 & $\pi-\pi$ \\
\hline PDC1 & HPhen & 298 & $\mathrm{BD}(2) \mathrm{C} 17-\mathrm{C} 21$ & 639 & $\mathrm{BD} *(2) \mathrm{C} 61-\mathrm{N} 118$ & 0.24 & $\pi-\pi$ \\
\hline $\mathrm{PDC} 2$ & HPhen & 180 & LP (3) O125 & 641 & BD*(2) C 62- C 63 & 0.08 & $\pi-\pi$ \\
\hline PDC2 & HPhen & 187 & LP (1) O129 & 639 & $\mathrm{BD}^{*}(2) \mathrm{C} 61-\mathrm{N} 118$ & 0.10 & $\pi-\pi$ \\
\hline $\mathrm{PDC} 2$ & HPhen & 200 & LP (3) O133 & 650 & BD*(2) C 66- C 67 & 0.10 & $\pi-\pi$ \\
\hline $\mathrm{PDC} 2$ & HPhen & 273 & $\mathrm{BD}$ (1) C 8-C 12 & 678 & $3 \mathrm{Cn}(1) \mathrm{C} 65-\mathrm{C} 68-\mathrm{C} 69$ & 0.06 & $\pi-\pi$ \\
\hline $\mathrm{PDC} 2$ & HPhen & 276 & $\mathrm{BD}(2) \mathrm{C} 8-\mathrm{O} 129$ & 639 & $\mathrm{BD}^{*}(2) \mathrm{C}$ 61- N118 & 0.28 & $\pi-\pi$ \\
\hline $\mathrm{PDC} 2$ & HPhen & 287 & $\mathrm{BD}(2) \mathrm{C} 12-\mathrm{C} 16$ & 678 & $3 \mathrm{Cn}(1) \mathrm{C} 65-\mathrm{C} 68-\mathrm{C} 69$ & 0.77 & $\pi-\pi$ \\
\hline $\mathrm{PDC} 2$ & HPhen & 287 & $\mathrm{BD}(2) \mathrm{C} 12-\mathrm{C} 16$ & 639 & BD*(2) C 61- N118 & 0.05 & $\pi-\pi$ \\
\hline $\mathrm{PDC} 2$ & HPhen & 287 & $\mathrm{BD}(2) \mathrm{C} 12-\mathrm{C} 16$ & 664 & BD*(2) C 72- N119 & 0.12 & $\pi-\pi$ \\
\hline PDC2 & HPhen & 307 & $\mathrm{BD}(2) \mathrm{C} 20-\mathrm{C} 24$ & 664 & $\mathrm{BD}^{*}(2) \mathrm{C} 72-\mathrm{N} 119$ & 0.29 & $\pi-\pi$ \\
\hline $\mathrm{PDC} 2$ & HPhen & 328 & $\mathrm{BD}$ (2) C 28- N113 & 678 & $3 \mathrm{Cn}(1) \mathrm{C} 65-\mathrm{C} 68-\mathrm{C} 69$ & 0.30 & $\pi-\pi$ \\
\hline $\mathrm{PDC} 2$ & HPhen & 340 & $\mathrm{BD}(2) \mathrm{C} 32-\mathrm{O} 137$ & 658 & BD*(2) C 70- C 71 & 0.11 & $\pi-\pi$ \\
\hline
\end{tabular}




\section{References}

1. Janiak, C., A critical account on pi-pi stacking in metal complexes with aromatic nitrogencontaining ligands. J. Chem. Soc., Dalton Trans. 2000, 3885-3896.

2. Mooibroek, T. J.; Gamez, P.; Reedijk, J., Lone pair- $\pi$ interactions: a new supramolecular bond? CrystEngComm 2008, 10, 1501-1515.

3. Caracelli, I.; Haiduc, I.; Zukerman-Schpector, J.; Tiekink, E. R. T., Delocalised antimony(lone pair)- and bismuth-(lone pair)... (arene) interactions: Surpamolecular assembly and other considerations. Coord. Chem. Rev. 2013, 257, 21, 2863-2879.

4. Steiner, T., C-H-O Hydrogen Bonding in Crystals. Crystallogr. Rev. 1996, 6, 1-51.

5. Steiner, T., The Hydrogen Bond in the Solid State. Angew. Chem. Int. Ed. 2002, 41, 48-76. 